\title{
Mining the Spatial-Temporal Characteristics of Population Flow and Material Flow Using Big Data
}

\author{
Xing Wang, ${ }^{1,2}$ Jianwan Ji, ${ }^{3,4}$ Biao Jin $\mathbb{D}^{1,2}$ Hongguang Chen, ${ }^{1}$ Shuhong Huang, ${ }^{5}$ \\ and Mingwei Lin ${ }^{1}$ \\ ${ }^{1}$ College of Mathematics and Informatics, Fujian Normal University, Fuzhou 350108, China \\ ${ }^{2}$ Digital Fujian Institute of Big Data Security Technology, Fuzhou 350108, China \\ ${ }^{3}$ Aerospace Information Research Institute, Chinese Academy of Sciences, Beijing 100094, China \\ ${ }^{4}$ University of Chinese Academy of Sciences, Beijing 100049, China \\ ${ }^{5}$ School of Geography, Nanjing Normal University, Nanjing 210023, China \\ Correspondence should be addressed to Biao Jin; jinbiao@fjnu.edu.cn
}

Received 27 May 2020; Revised 24 July 2020; Accepted 16 September 2020; Published 29 September 2020

Academic Editor: Caroline Mota

Copyright ( 92020 Xing Wang et al. This is an open access article distributed under the Creative Commons Attribution License, which permits unrestricted use, distribution, and reproduction in any medium, provided the original work is properly cited.

\begin{abstract}
Population flow and material flow are the core elements of the "space of flows." Studying the temporal and spatial distribution characteristics of these two types of flows (TToF) can provide us a greater understanding of the research area. Most of the existing works related to TToF only focus on exploring the difference characteristics of one of the members of TToF in a certain time or space scale in the research area. Different from these related works, the spatial-temporal characteristics of the population flow and material flow in Taiwan Province and the spatial-temporal autocorrelation of Taiwan's expressway network are explored by means of multimembership and layer-by-layer refinement. The research work carried out in this paper includes the following: (1) studying the differentiated characteristics of the TToF in different time units; (2) studying the spatial differences among each type of the TToF under different scales; (3) dividing both the population flow and material flow into two subtypes and then analyzing the temporal variation characteristics of the four subtypes of flows; and (4) studying the global and local spatial-temporal autocorrelation of Taiwan's expressway network. The results show the following. (1) The spatial-temporal differentiation characteristics of the TToF are obvious in different time units and on different scales. (2) The contribution of the population flow to the TToF in flow quantities is far greater than that of the material flow. (3) The population flow and material flow are dominated by the "minority population flow" and "small-scale material flow," respectively. (4) Meanwhile, in Taiwan's expressway network, there is a significant spatial-temporal positive correlation mainly reflected in the spatial first-order adjacent road sections.
\end{abstract}

\section{Introduction}

With the rapid development of information technology, the influence of a fixed geographical location on production and organization has gradually weakened. Power and function are organized in the flows. The dominant form of space is no longer the traditional space ("space of places") but rather is a new space ("space of flows"). This new space transcends the boundaries of time and space, playing a guiding role in the development of regions' spatial patterns in the future. With the help of the interaction of various "flows" in the space, it extends from its interior to many fields of human production and life. The core elements of the "space of flows" include population flows, material flows, capital flows, and information flows. Among these flows, information flows, material flows, and population flows are often mentioned and discussed. However, the data associated with the information flows are very difficult to obtain because it usually involves the private information of units or individuals. According to the literature we collected, we determine that the current studies on flows are more focused on population flows and material flows.

Population mobility is an extremely complex research topic. Many researchers can only choose some more specific 
subtopics for research, such as studying the impact of population mobility on regional economic development $[1-3]$, exploring the relationship between population mobility and urbanization development [4-6], and mining the spatial pattern and formation mechanism of population mobility [7-10]. Based on the interprovincial migration data during the period 1985-2010, literature [7] used bicomponent trend mapping technique with every five years as a time interval to analyze the intensity and change pattern of in-migration and out-migration from 1985 to 2010. Literature [8] analyzed the spatial-temporal characteristics and influencing factors of population changes in Beijing-TianjinHebei (BTH) region from the two aspects of population distribution patterns and population flow in long and short periods. Their work enlightens us that in order to understand a problem or phenomenon more thoroughly, we can analyze it from many different perspectives.

The process of population mobility is often accompanied by the flow of information, material, capital, and technology. The mobility of a population among different regions leads to the dynamic adjustment of relevant resources, such as the labor force, talent, technology, and so on [11]. The impact of this dynamic adjustment is often complex. For example, a labor force inflow greatly supports and speeds up the infrastructure construction in the destination areas. In contrast, a labor force outflow also causes a large number of old people and children to be left behind and brings a series of social problems. As another example, the mobility of talents from backward areas to developed areas will further increase the gap in the comprehensive strength and competitiveness of two types of areas. In contrast, the gap between the two types of areas may gradually become narrow. Furthermore, long-term or regular large-scale population migration will not only affect the decision making of relevant government departments but also stimulate the construction of the infrastructure (such as housing, medical care, education, and so on) closely related to the floating population in the relevant regions [12].

The material flow is the most important flow in an economic system. It influences regional economic growth by affecting both supply and demand. It is not only conducive to the deepening of the regional division of labor and specialized production and the effective input of elements, but it can also expand output demand [13]. Currently, management scholars are committed to studying the patterns of material flows $[14,15]$ and discussing the location planning of material flow centers [16, 17]. Economists are committed to mining the relationship between the material flow and economic development $[18,19]$. From the geographical perspective, the related research on material flows mainly focuses on the discovery of the spatial patterns and the evolutionary development of a material flow enterprise or the regional logistics chain [20-24]. The method of data envelopment analysis (DEA) and exploring statistics has been used by literature [23] to analyze the whole evolution trend, structure, and spatial pattern's evolutionary process and its evolutionary mechanism of Yangtze River delta city logistics development efficiency from 2002 to 2011 based on the input-output index of city logistics. Literature [24] takes the major 22 ports along the Yangtze River as the research object and develops a comprehensive evaluation index system of port logistics development. Then, it analyzes the spatiotemporal evolution characteristics and mechanism of port logistics development along the Yangtze River, by using the methods of Gini coefficient and standard deviational ellipse. Their work has extended the application of some classical correlation analysis methods.

To sum up, population mobility is a complex and important research topic. Its process is often accompanied by the flow of information, material, capital, and technology. The analysis of population mobility is of great significance to the development of economy and the formulation of policies. Most of the existing related literatures study population mobility from a certain dimension or local subproblem with specific research purposes but lack multidimensional perspective of exploration and research. Material flow is an important manifestation of economic activities. The study of material flow is not only the need of theoretical research on industrial economy but also the practical need of the development of logistics industry. At present, most of the literatures focus on the research of logistics mode and logistics technology. The research of material flow needs to be further expanded and deepened. This paper is committed to providing multidimensional analysis methods for the study of population flow and material flow. Taiwan Province and its electronic toll collection (ETC) data are taken as the research area and fundamental experiment data, respectively.

Taiwan Province, with its superior geographical position, is located at the southeast gateway of China as an important channel to the Pacific Ocean. The study of the population flow and material flow in Taiwan Province can help us to better understand the province. At present, the main purpose of relevant studies on the population flow in Taiwan Province is to determine the long-term migration trend of the population on and between the islands [25-27], while the research on the material flow focuses on its logistics policy and competitiveness [28-30].

Different from the starting point of the existing related work [25-30], this paper focuses on the research of the spatial-temporal differentiation characteristics of the population flow and material flow (referred to "two types of flows, TToF") in Taiwan Province. In addition, different from those literatures which analyze the spatial-temporal differentiation characteristics of TToF in a specific geographical area and only in a certain time scale and space scale, we adopted the multimembership and layer-by-layer refinement methods for conducting our analysis work. The research work carried out in this paper mainly includes the following: (1) studying the differentiation characteristics of the TToF in different time units (hourly, daily, and monthly), (2) studying the spatial differences of each type of the TToF under different scales (detection sites, road sections, counties, and cities), and (3) studying the global and local spatial-temporal autocorrelation of Taiwan expressway network. Our experiments and analysis are mainly based on the ETC data of Taiwan Province in 2017. Our work would help to provide a better understanding of the travel habits of 
the people and the mode of the material flow in Taiwan Province.

\section{Experimental Data and Methods}

In this paper, we take Taiwan Province as the research area and the ETC data of its expressway as the fundamental experimental data. Then, the spatial-temporal characteristics of the population flow and material flow in Taiwan Province and the spatial-temporal autocorrelation of Taiwan's expressway network are explored by means of multimembership and layer-by-layer refinement.

2.1. Overview of the Research Area. Taiwan Province $\left(21^{\circ} 53^{\prime} \sim 25^{\circ} 18^{\prime} \mathrm{N}, 120^{\circ} 08^{\prime} \sim 122^{\circ} 01^{\prime} \mathrm{E}\right)$ is located on the southeast coast of China, bordering the Pacific Ocean to the east, Ryukyu Islands to the northeast, and Fujian Province across the Taiwan Strait to the west. Its north-south longitudinal length is approximately $395 \mathrm{~km}$, its east-west width is approximately $144 \mathrm{~km}$, its total area is approximately 36000 square kilometers, and its total population is approximately 23.54 million (the ratio of men and women is close to $1: 1$ ). In terms of landforms, approximately $70 \%$ of the province is mountainous and hilly, and the plains are mainly concentrated on the west coast, with great changes in terrain and altitude. It is located at the junction of tropical and subtropical climates (subtropical monsoon climate to the north of the Tropic of Cancer and tropical monsoon climate to the south). More detailed information about Taiwan Province can be obtained from [31]. Figure 1 shows the administrative map of Taiwan Province.

The experimental data used in this paper are the ETC data of Taiwan expressway in 2017. The data cover 16 counties and cities in Taiwan except Chiayi City, Hualien County, and Taitung County.

2.2. Experimental Data. The ETC data of the expressway in Taiwan Province are our fundamental experimental data. The original data are recorded by the detection sites on the expressway and can be obtained from the official website. Considering the research purpose of this paper, it is necessary to further process the original data.

2.2.1. Data Sources. At present, there are 331 detection sites along Taiwan expressway. They divide Taiwan expressway into 481 detection areas. The location of each detection site and the number of detection sites in each county and city are shown in Figure 2 and Table 1, respectively.

In this paper, we take the M05A files from the ETC data of Taiwan expressway in 2017 as the fundamental experimental data. The data in this file are recorded every 5 minutes, and thus 253,017,045 records are obtained. Each record contains six fields, such as TimeInterval (report generation time), GantryFrom (no. of upstream detection sites), GantryTo (no. of downstream detection sites), VehicleType (vehicle's type), SpaceMeanSpeed (average speed), and VehicleCount (traffic volume for each vehicle type in the current time interval). The vehicle types are as follows: 31 for minibuses, 32 for pickup trucks, 41 for buses, 42 for trucks, and 5 for coupling vehicles.

2.2.2. Data Preprocessing. To find the clear spatial-temporal differences of the population flow and material flow in Taiwan Province, we first obtain the latitude and longitude information of the 331 detection sites and import the data in the relevant record tables into an SQL Server 2008 database after merging them on a monthly basis. After that, extra fields such as "hour, week, and county/city of starting detection site and ending detection site" are added to each record. The detailed steps of data preprocessing are shown in Table 2 .

2.3. Research Methods. ETC data are time series in nature. The times generated by the records can be used to analyze the temporal differences of the traffic volume, while the spatial differences in the traffic volume can be analyzed by combining the longitude and latitude information of detecting sites with the administrative divisions of Taiwan Province.

After analyzing the spatial-temporal differences of the traffic volume, this paper further studies the spatial-temporal autocorrelation of Taiwan's expressway network. Considering that the overall distribution of the phenomenon is described by the global analysis index, it is not only difficult to detect the correlation patterns of different regions, but it may also conceal some local heterogeneity by using only one single value to reflect the overall situation. Therefore, this paper analyzes both the global and the local spatial-temporal autocorrelation of Taiwan's expressway network.

The multimembership and layer-by-layer refinement analysis method is adopted to conduct our research work. Our research framework is shown in Figure 3.

Seen from Figure 3, the analysis idea of multimembership and layer-by-layer refinement mainly embodies the following aspects. (1) Classifying the experimental data into two parts according to the actual purpose of vehicles, one for the analysis on population flow and the other for material flow. (2) Both of the population flow and material flow are divided into two subtypes, and then four kinds of flows are obtained. (3) For the four kinds of flows, we analyzed their differences in the temporal characteristics in different time units (hourly, daily, and monthly). (4) For the TToF, we studied their spatial differences under different scales (detection sites, road sections, counties, and cities). (5) For the TToF, we explored not only their global spatial-temporal autocorrelation but also local spatial-temporal autocorrelation.

2.3.1. Spatial and Temporal Difference Analysis Method. To make the analysis results more accurate and make the best use of the experimental data, according to the actual purposes of vehicles, the records corresponding to vehicle types 31 and 41 in the experimental data are regarded as population flow data while the records corresponding to vehicle types 5,32 , and 42 are regarded as material flow data. In addition, to achieve a better understanding of Taiwanese travel habits and 


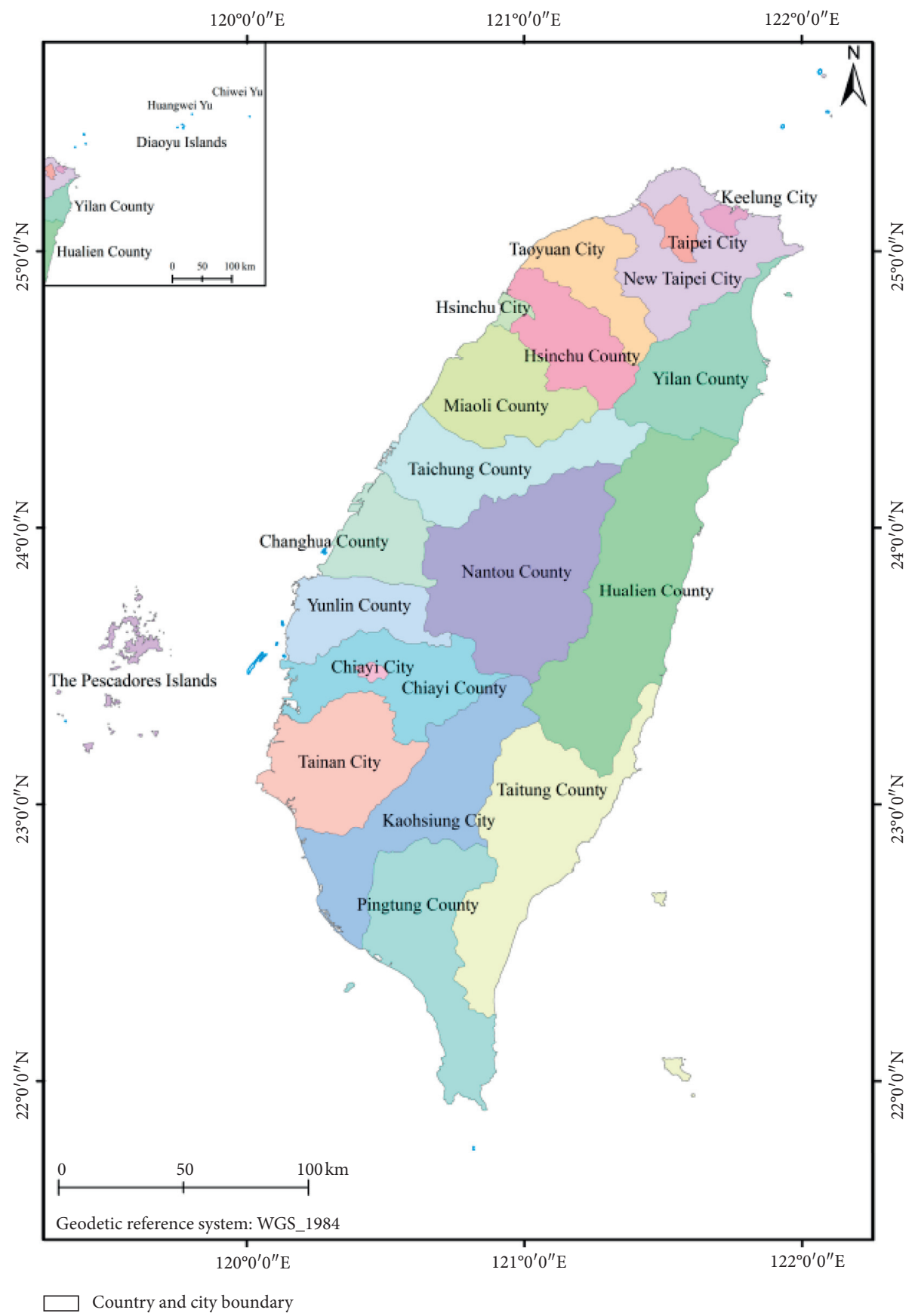

Figure 1: Administrative map of Taiwan.

the material flow patterns of Taiwan, we further subdivide the TToF into four subtypes: (1) considering the records corresponding to vehicle type 31 as minority population flow; (2) considering the records corresponding to vehicle type 41 as mass population flow data; (3) considering the records corresponding to vehicle type 32 as small-scale material flow data; and (4) considering the records corresponding to vehicle types 5 and 42 as large-scale material flow data.

We seek to determine the spatial-temporal differentiation characteristics of the TToF in Taiwan Province. By adopting the means of multimembership and layer-by-layer refinement methods, we not only study the temporal differences of different types of flows in different units (monthly, daily, and hourly) but also study the spatial differences of different types of flows in different units (detection sites, road sections, and counties or cities).

2.3.2. Spatial-Temporal Autocorrelation Analysis Method. In this paper, the ST-ACF (spatial-temporal autocorrelation function) proposed by Pfeifer et al. $[32,33]$ and the CCF (cross correlation function) proposed by Box and Jenkins [34] were used to analyze the global spatial-temporal autocorrelation and local spatial-temporal autocorrelation of Taiwan's expressway network, respectively. The ST-ACF can be used to measure the correlation between samples with an 


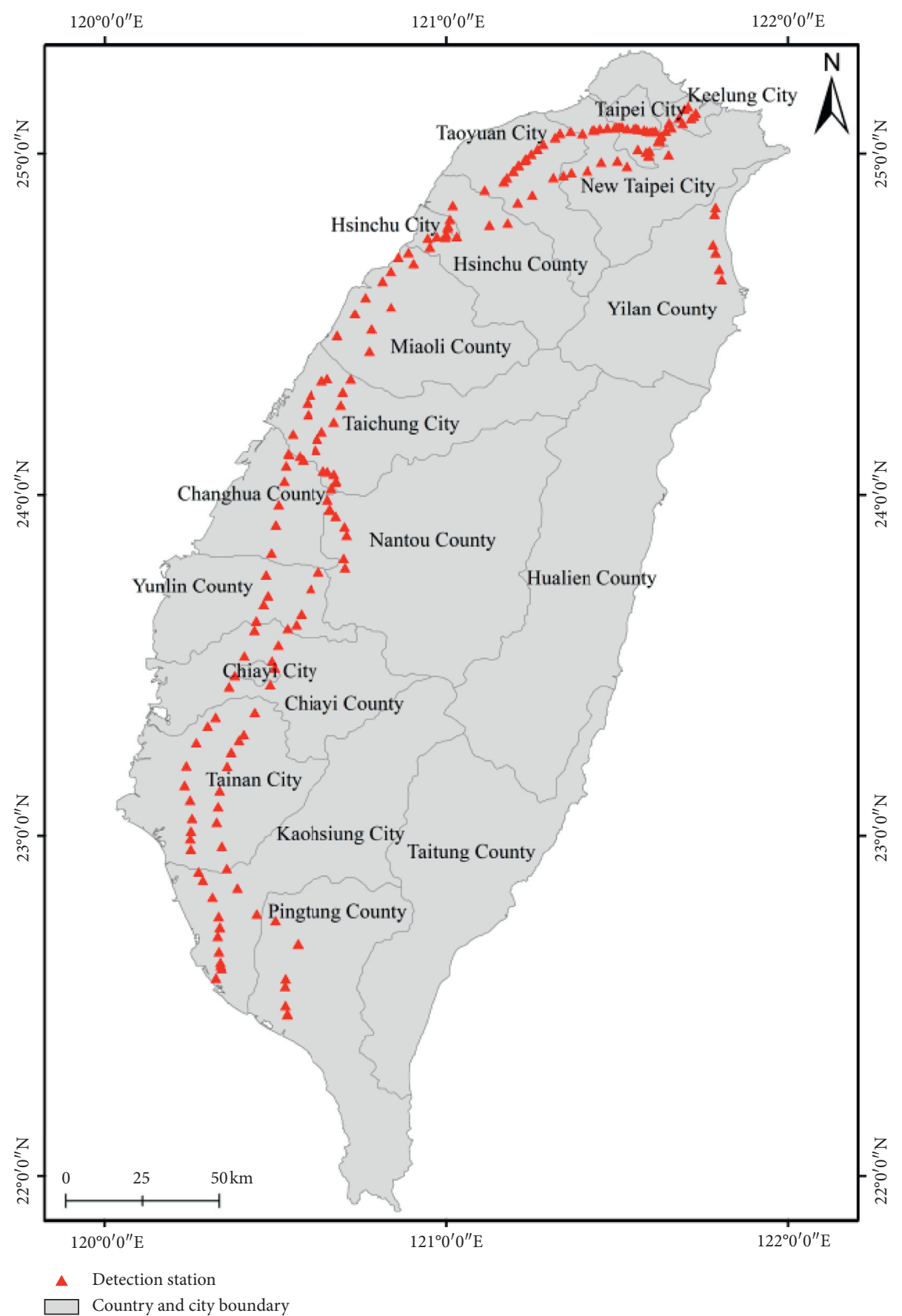

Figure 2: Distribution map of the detection sites.

arbitrary spatial delay and time delay for the two aspects of space and time, respectively, while the CCF can be used to measure the correlation between two regional samples over a specified time delay. Some scholars have proved that CCF can be used to measure the spatial-temporal autocorrelation among road network sections [35].

The calculation formula for ST-ACF [32] is as follows:

$$
\rho_{h 0}(s)=\frac{r_{h}(s)}{\sqrt{\sigma_{h}(0) \sigma_{0}(0)}}=\frac{\operatorname{cov}\left(W^{h} Z(t), W^{0} Z(t+s)\right)}{\sqrt{\operatorname{var}\left(W^{h} Z(t)\right) \cdot \operatorname{var}\left(W^{0} Z(t+s)\right)}},
$$

where $\rho_{h 0}(s)$ is the spatial-temporal autocorrelation coefficient; $h$ and $s$ stand for the space delay and time delay, respectively; $r_{h}(s)$ stands for the sample covariance with a spatial delay of $h$ and a time delay of $s ; \sigma_{h}(0)$ represents the sample variance with a spatial delay of $h$ and a time delay of $0 ; W^{h}$ represents the spatial weight matrix whose spatial delay is $h$ (when $h=0$, this weight matrix is an identity matrix); and $Z(t)$ and $Z(t+s)$ stand for the specific property values of research unit $Z$ at times $t$ and $(t+s)$, respectively. In our experiment, $Z(t)$ stands for the traffic 
TABLE 1: Quantity distribution of the detection sites in each county and city.

\begin{tabular}{lc}
\hline County/city & Sites \\
\hline Kaohsiung City & 24 \\
Keelung City & 11 \\
Chiayi County & 16 \\
Miaoli County & 22 \\
Nantou County & 14 \\
Pingtung County & 12 \\
Taipei City & 23 \\
Tainan City & 40 \\
Taichung City & 36 \\
Taoyuan City & 30 \\
New Taipei City & 37 \\
Hsinchu City & 6 \\
Hsinchu County & 16 \\
Yilan County & 10 \\
Yunlin County & 18 \\
Changhua County & 16 \\
\hline
\end{tabular}

volume at time $t$ of road section $Z$ and $s=5$ since the ETC system generates a record every five minutes.

Let $X$ and $Y$ be two time series. Then, their spatialtemporal correlation coefficients under the specified time delay $s$ and based on the CCF function [34] can be calculated as follows:

$$
r_{X Y}(s)=\frac{E\left[\left(X_{t}-\bar{X}\right)\left(Y_{t+s}-\bar{Y}\right)\right]}{\sqrt{\sigma_{X} \sigma_{Y}}},
$$

where $\bar{X}$ and $\bar{Y}$ are, respectively, the average values of $X$ and $Y$ and $\sigma_{X}$ and $\sigma_{Y}$ are the variances of the two respective sequences.

To calculate the values of the ST-ACF and CCF, the spatial weight matrix needs to be substituted into the formulas. In this paper, the spatial first-order adjacency matrix and spatial second-order adjacency matrix of each road section are used as the weight matrix following the practice of literature [36]. The first-order and second-order adjacency relations of each section in the road network are defined as follows.

(i) First-order adjacency of a road section: if the end point of section $\mathrm{A}$ is exactly the starting point of section $B$, then sections $A$ and $B$ are said to be firstorder adjacency to each other.

(ii) Second-order adjacency of a road section: if section $\mathrm{B}$ is first-order adjacent to both section $\mathrm{A}$ and section $\mathrm{C}$, then sections $\mathrm{A}$ and $\mathrm{C}$ are second-order adjacency. If sections A and B have the same starting or ending points, sections $\mathrm{A}$ and $\mathrm{B}$ are second-order adjacent to each other.

According to the above definition, the first-order and second-order adjacency matrices of the road network shown in Figure 4(a) are given in Figures 4(b) and 4(c), respectively.

Then, the first-order and second-order adjacency matrices of Taiwan's expressway network which consists of 331 detection sites and 481 road sections can be generated. The two matrices will be denoted as $W^{1}$ and $W^{2}$, respectively.

\section{Analysis and Discussion}

Different kinds of flows are classified by the vehicle type in each record. By this way, two broad types (population flow and material flow) and four subtypes (minority population flow, mass population flow, small-scale material flow, and large-scale material flow) are obtained. Then, both the differentiated characteristics of each kind of flow in different time units and the spatial differences among each type of the TToF under different scales are analyzed. Moreover, we also discussed the global and local spatial-temporal autocorrelation of Taiwan's expressway network.

3.1. Analysis of the Differences in the Temporal Characteristics of the Total Circulation of the TToF. As mentioned above, the differentiated characteristics of the TToF are analyzed in different time units (monthly, daily, and hourly).

\subsubsection{Differences in the Monthly Characteristics of the TToF.} Since each data table is built monthly during data preprocessing, the total circulation of the TToF in each month can be obtained by querying the statistics based on the vehicle types. For the different days of each month, in order to more reasonably reflect the difference in the monthly flows, we first calculate the daily average flow of each month and then use the min-max standardization method to normalize the relevant data. The results are shown in Figure 5.

Figure 5 shows the following. (1) August is the most active period of Taiwan's material flow, and its tide period appears in June. According to the data provided by the official website of http://www.tianqihoubao.com/lishi, in June 2017, there are more than 20 rainy days on average in the major counties and cities of Taiwan Province, and there are even several days of heavy rain and rainstorms. Bad weather affected the traffic volume on the expressway, which has greatly reduced the material flow. (2) In January and February, the population flow is the most active. The main reason is that there are many important festivals such as New Year's Day, the Spring Festival, Valentine's Day, the Lantern Festival, and Peace Memorial Day in January and February. They are bound to stimulate the growth of the population flow. Influenced by bad weather, June is also a low population flow period. (3) Regarding the overall change trends, the change trend of the monthly mean flow of material flow is much gentler than that of the population flow. (4) The contribution of the population flow to total circulation is far greater than that of the material flow, which shows that its monthly change trend is very consistent with that of the total circulation of the TToF. The correlation between the blue curve and green curve is approximately $98.74 \%$, while the correlation between the yellow curve and green curve is only approximately $34.89 \%$.

\subsubsection{Differences in the Daily Characteristics of the TToF.} By querying the statistics according to the new field "week" added in the preprocessing process and the vehicle types, the 
TABLE 2: The data preprocessing steps.

Step 1 Obtain the longitudes and latitudes of the 331 detection sites and the counties and cities where they are located in from Google maps.

Step 2 For the convenience of the classification and statistics, use the command "copy*.csv reslutSave.csv" to merge the record data in the M05A file by month to form twelve csv files.

Step 3 Import the twelve CSV files into an SQL server database to obtain the twelve record tables.

Additionally, for the convenience of the classification and statistics, add fields such as [hour, week, and administrative region where

Step 4 the origin and destination sites are located] to each record. The values of these added fields are set according to the information from Step1.

Write and execute statements or commands [select, group by, order by, count (...)] for obtaining the statistical information about Step 5 the traffic volume of the different type of vehicles at different sites, in different road sections and in different time intervals.

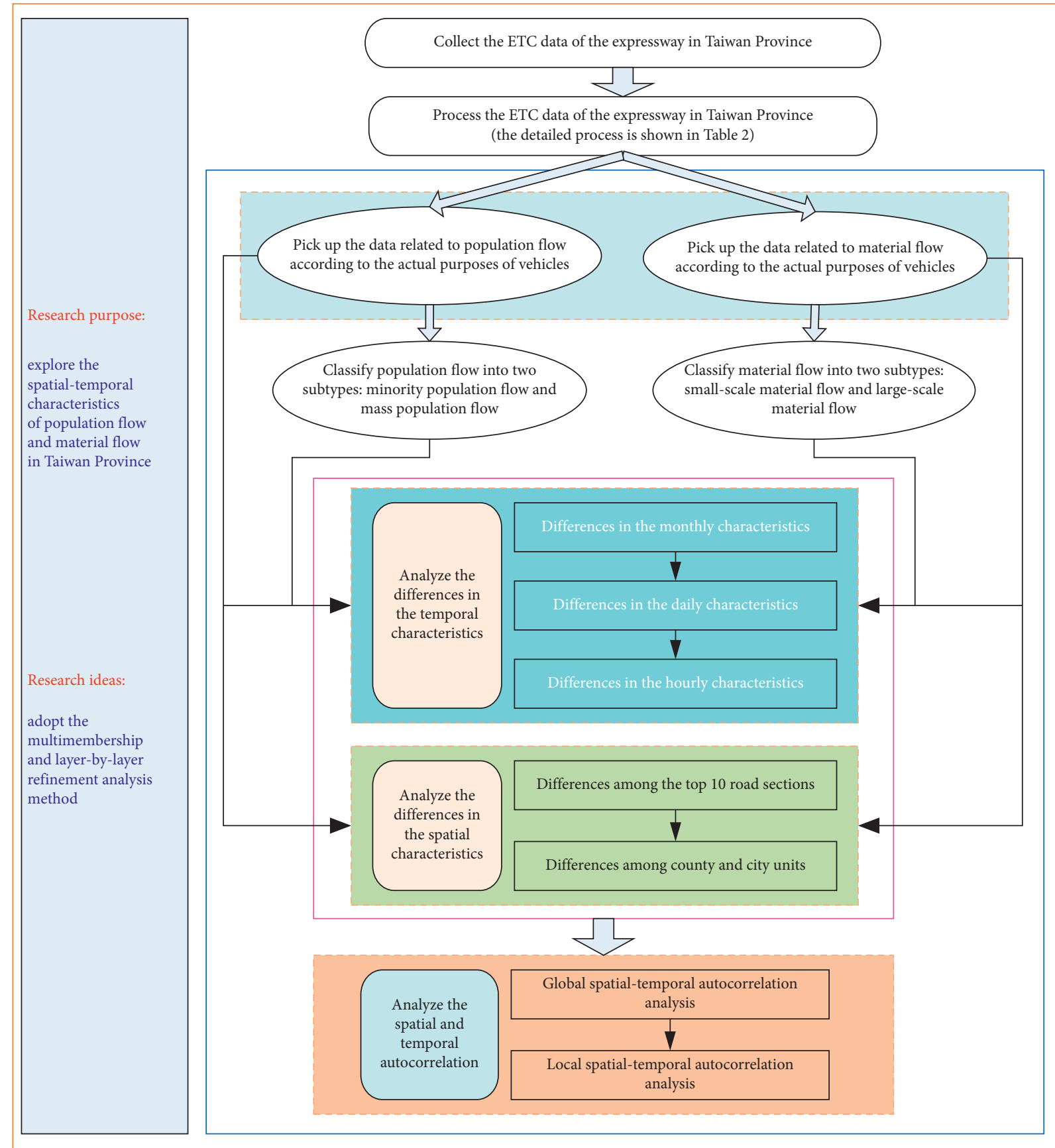

FIgURE 3: Research framework of our work. 


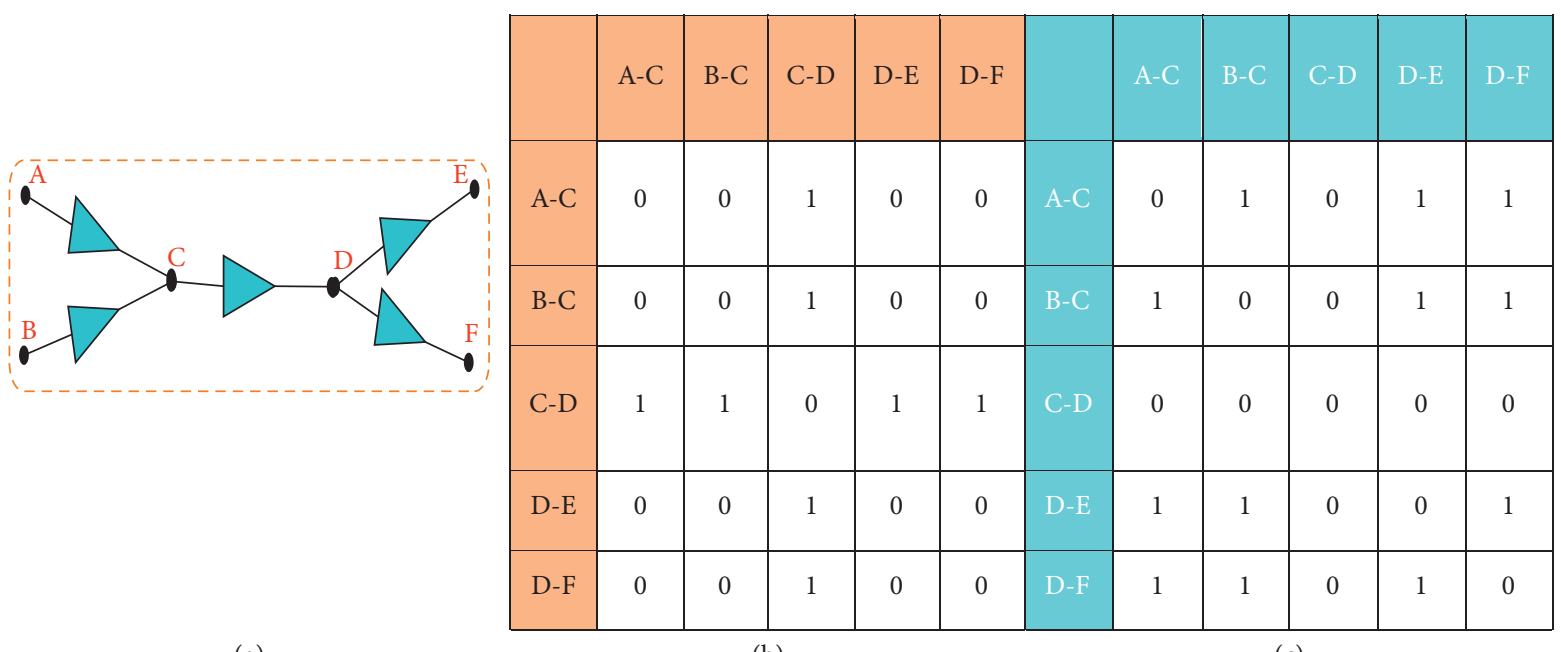

(a)

(b)

(c)

Figure 4: An instance of a road network and its adjacency matrices. (a) An instance of a road network. (b) The first-order adjacency matrix of the instance. (c) The second-order adjacency matrix of the instance.

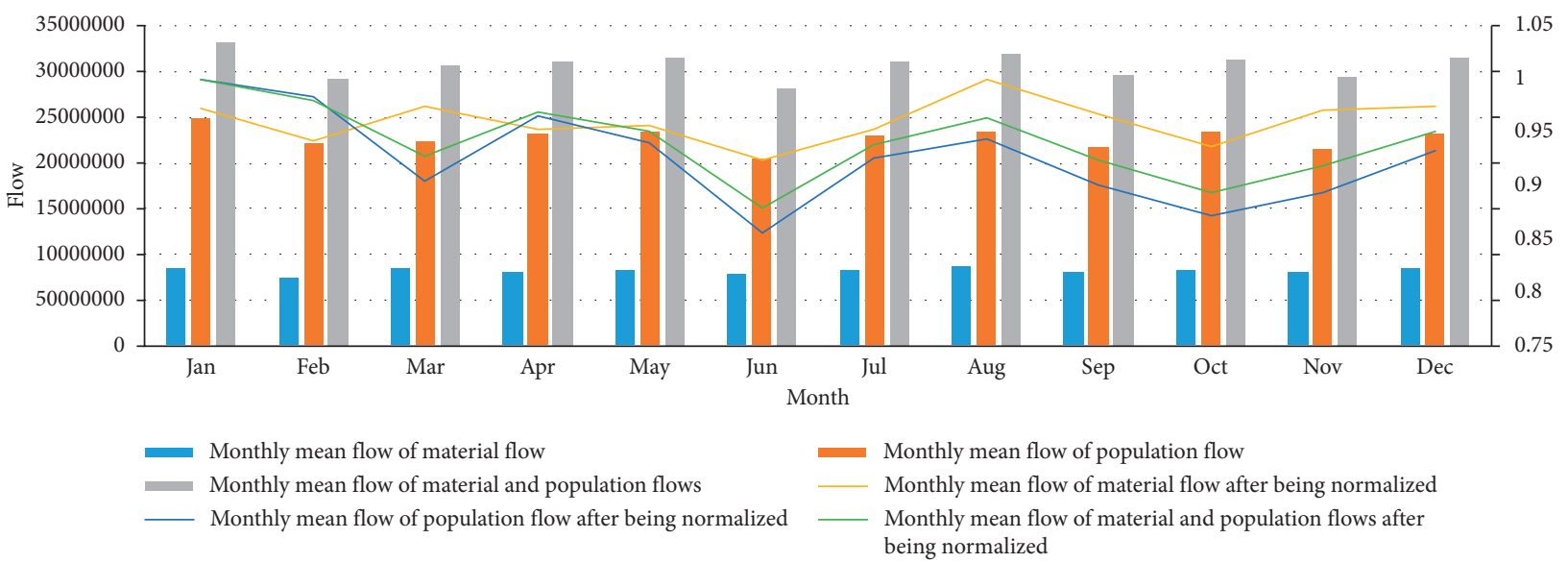

Figure 5: Monthly change trend of the monthly mean flow of the TToF.

total circulation volume of the TToF on the different days of the week can be obtained. Since the number of Mondays to Sundays is not exactly the same throughout 2017 (Monday to Saturday occur 52 times while Sunday occurs 53 times), first, we calculate the average circulation of on each day in a week, and then normalize the results. The relevant results are shown in Figure 6.

The following can be seen from Figure 6. (1) On weekdays, the daily material flow shows an overall upward trend, and the peak appears on Friday. (2) On weekdays, the daily population flow decreases from Monday to Thursday, but increases significantly on Friday. (3) The population flow on weekends is significantly higher than that on weekdays. One reason for this phenomenon is that Taiwan's infrastructure, such as its roads and corresponding services, is perfect. In contrast, the material flow drops sharply on weekends. Selectively avoiding the peak period of public travel can save transportation costs for logistics companies. (4) From the perspective of the total TToF, the total flow on weekends is much higher than that on weekdays because the contribution of the population flow to the total flow is much greater than that of the material flow. The percentage of the population flow to the total flow on weekdays is approximately $70.10 \%$ while that on weekends is approximately $81.08 \%$.

3.1.3. Differences in the Hourly Characteristics of the TToF. By querying the statistics according to the new field "hour" added in the preprocessing process and the vehicle types, the total circulation volume of the TToF on different hours of a day can be obtained. After normalizing the total population flow, the total material flow, and the sum of the two in each hour, the hourly change trend of the TToF is obtained, as shown in Figure 7.

The following can be seen from Figure 7. (1) In a day, the most active periods of the material flow are 9:00-11:00 and 14:00-15:00 with peak points at 10:00 and 15:00, respectively. (2) The most active time periods of the population flow are 8:00-11:00 and 16:00-18:00 with the peak points at 


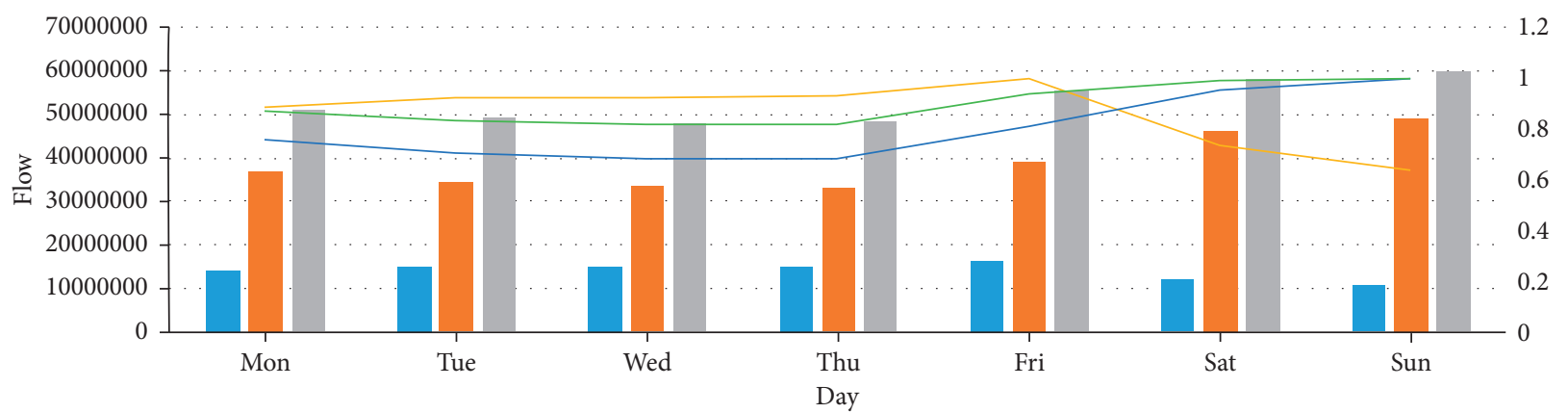

Daily mean flow of material flow

Daily mean flow of material and population flows

_ Daily mean flow of population flow after being normalized

Daily mean flow of population flow Daily mean flow of material flow after being normalized

_ Daily mean flow of material and population flows after being normalized

Figure 6: Daily change trends of the daily mean flows of the TToF.

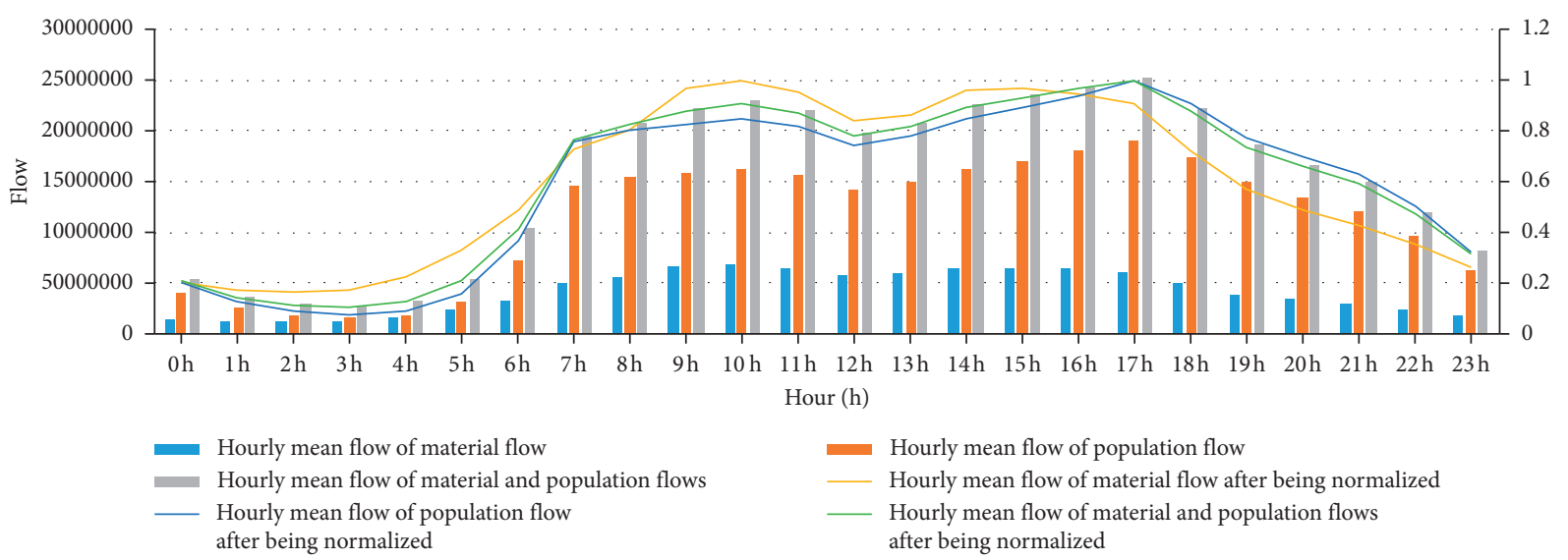

FIgURE 7: Hourly change trends of the hourly mean flows of the TToF.

10:00 and 17:00, respectively. (3) 00:00-5:00 is the period with the lowest traffic pressure on the expressway. This period is the valley of the TToF. 17:00 is the busiest time of a day on the expressway. (4) On the whole, Taiwan's population flow and material flow increase rapidly starting at 6: 00 and reach the first peak at 10:00. There is a slight decline from 10:00 to 12:00, and then it rises again. The second and third peaks of the TToF are at 15:00 and 17:00, respectively. After 17:00, the both components of the TToF rapidly decrease. We can find that the hourly change trends of the TToF coincide with Taiwanese work and living habits.

\subsection{Analysis of the Differences in the Temporal Characteristics} of the Minority Population Flow and Mass Population Flow. To study Taiwanese travel habits more deeply and make full use of the data in each field of the original data, we further divide the population flow into the "minority population flow" (vehicle type 31) and the "mass population flow" (vehicle type 41) according to vehicle types in the original data. Then, we analyze the time characteristics of the two kinds of population flows and find the differences between them. The processing methods of the two kinds of population flow data are consistent with those of the TToF data in the first subsection of this section.
3.2.1. Differences in the Monthly Characteristics of the Two Kinds of Population Flows. The monthly mean flow and the monthly change curves of the two kinds of population flows are normalized and shown in Figure 8.

The following can be seen from Figure 8. (1) January to February and July to August are the peak periods of the minority population flow with the troughs in June and November. (2) In contrast to the minority population flow, January to February and July to August are the troughs of the mass population flow, March to May and October to December are the active periods, and the highest peak time appears in November. The main reason for this phenomenon is that January to February and July to August are the months with the lowest and highest average temperatures in Taiwan, respectively. During these time periods, people tend to travel by car. On the whole, the monthly mean change trends of the mass population flow is gentler than that of the minority population flow. The contribution of the minority population flow to the total population flow is far greater than that of the mass population flow.

3.2.2. Differences in the Daily Characteristics of the Two Kinds of Population Flows. The daily change trends of the mean minority population flow and mass population flow in a week are shown in Figure 9. 


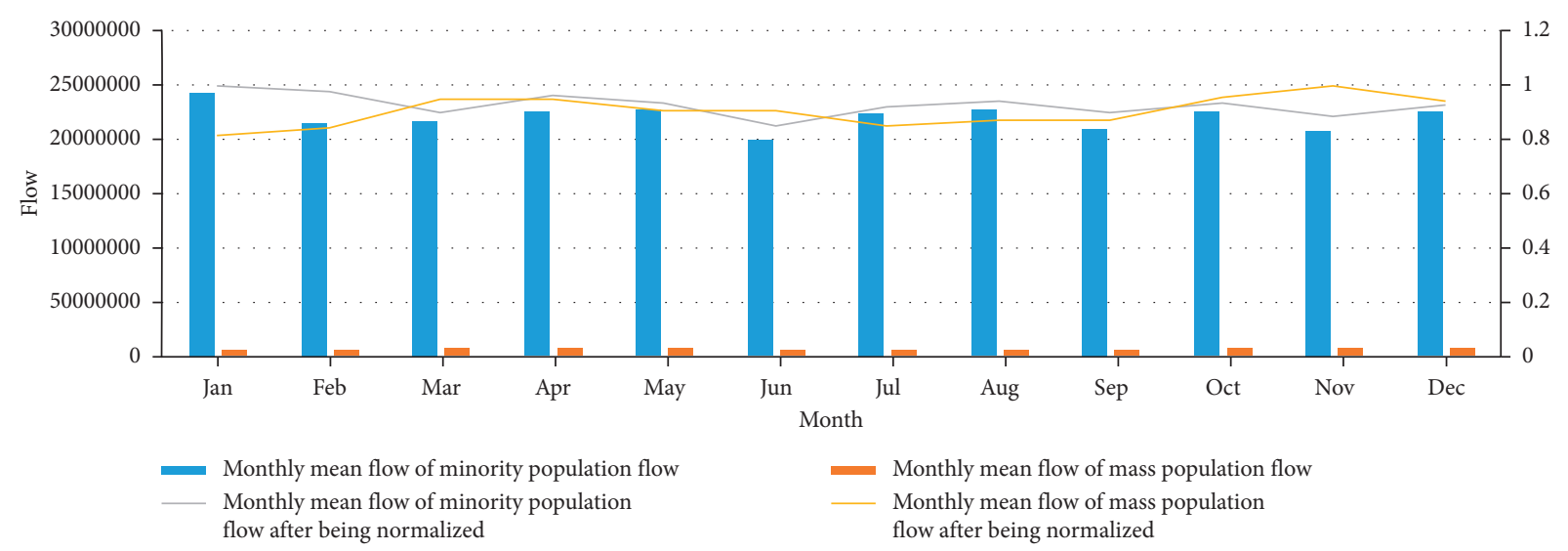

Figure 8: Monthly change trends of monthly mean flows of the two kinds of population flows.

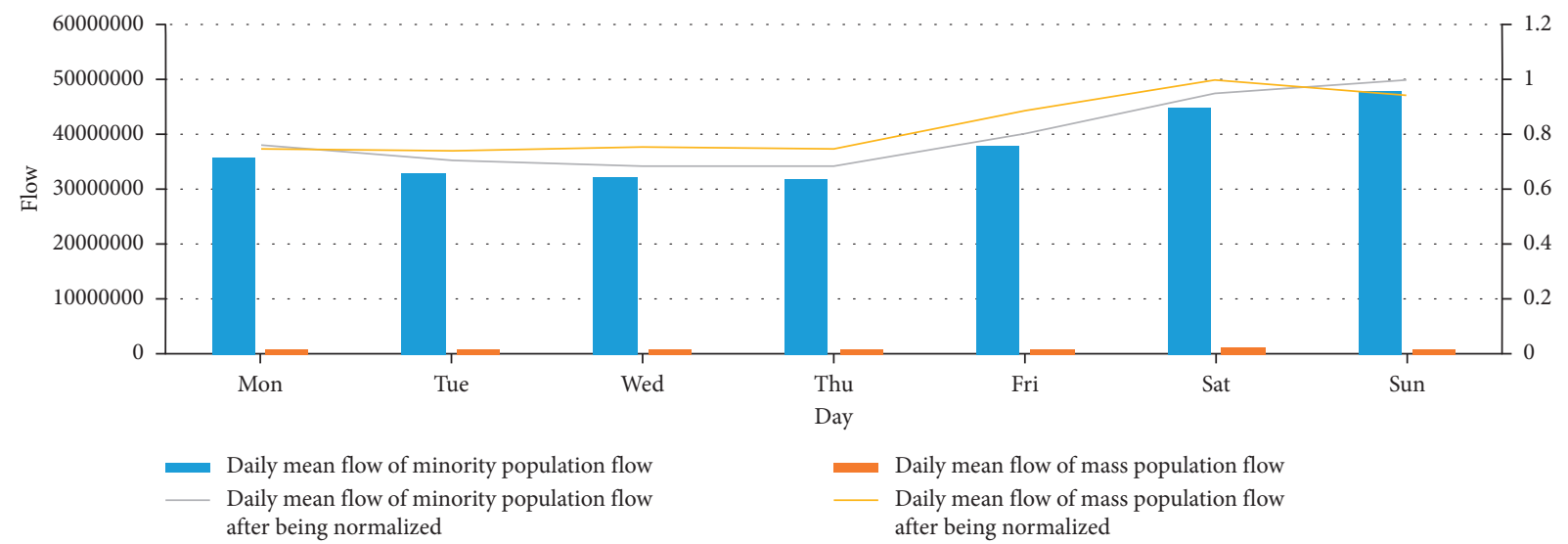

Figure 9: Daily change trends of daily mean flows of the two kinds of population flows.

The following is shown in Figure 9. (1) The minority population flow shows a slight downward trend from Monday to Thursday, and it gradually increases in the next three days, reaching its peak on Sunday. (2) The mass population flow shows an overall upward trend from Monday to Saturday and has peaks on Saturday and declines slightly on Sunday. Combining (1) and (2), on weekdays, Taiwanese prefer to take public transport. It also reflects Taiwan's perfect transportation infrastructure and services. In addition, on the whole, the circulation volume of these two kinds of population flows on the weekends is higher than that on weekdays.

3.2.3. Differences in the Hourly Characteristics of the Two Kinds of Population Flows. Figure 10 shows the hourly change trends of the mean minority population flow and mass population flow.

Figure 10 shows us the following. (1) The minority population flow and mass population flow in each hour show double peak characteristics. (2) The two peaks of the two kinds of population flows are not the same. The first peak of the mass population flow appears at 8:00 while the first peak of minority population flow appears at 10:00. 17:00 is the other peak of the two kinds of population flows.
3.3. Analysis of the Differences in the Temporal Characteristics of the Small-Scale Material Flow and Large-Scale Material Flow. Correspondingly, in order to further understand the pattern of the material flow in Taiwan Province, we divide the material flow into the small-scale material flow (vehicle type 32) and large-scale material flow (vehicle types 5 and 42) according to the vehicle types in the original data. Then, the temporal characteristics of the two kinds of material flows are analyzed to find the differences between them. The data preprocessing of these two kinds of material flows is also consistent with that of the TToF in the first subsection of this section.

3.3.1. Differences in the Monthly Characteristics of the Two Kinds of Material Flows. The monthly mean flows and the monthly change curves of the two kinds of material flows are normalized and shown in Figure 11.

The statistical results show the following (Figure 11). (1) March, June, August, and November are the active periods of the large-scale material flow while its troughs are from January to February. (2) The active periods of the small-scale material flow appear in January and August, and June is its trough. We think one of the main reasons for (1) and (2) is that the discount season in Taiwan varies with different products. (a) It is a good 


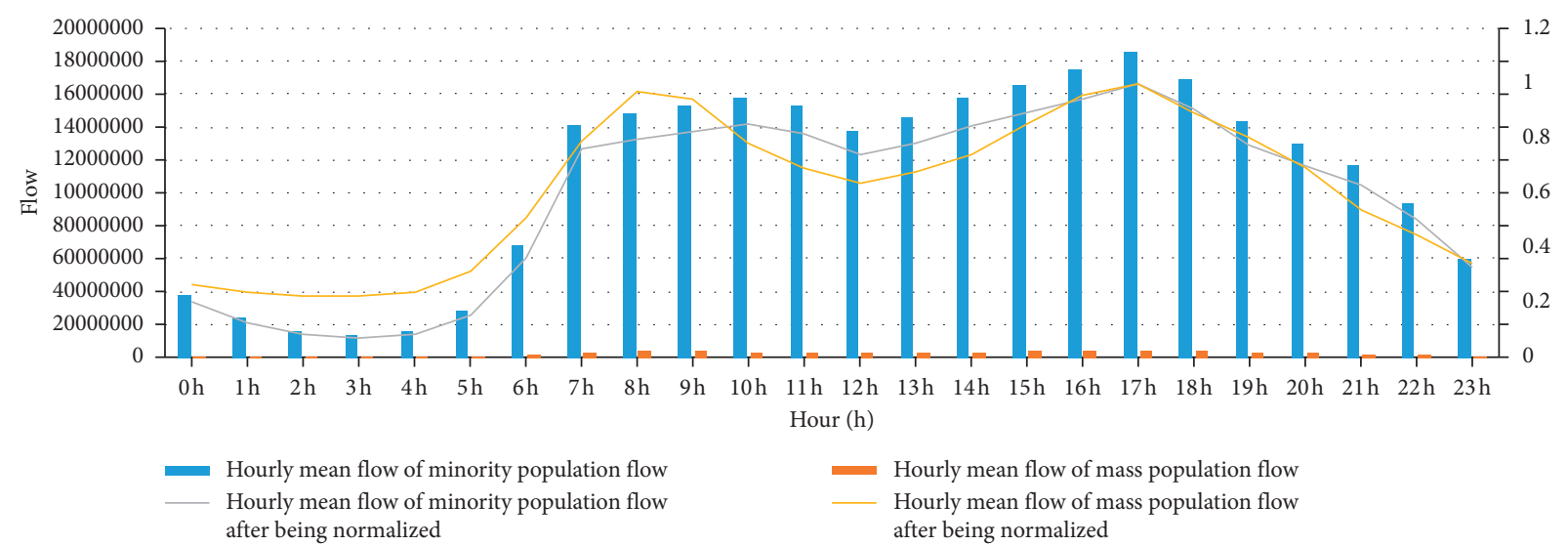

FIgURE 10: Hourly change trends of the hourly mean flows of the two kinds of population flows.

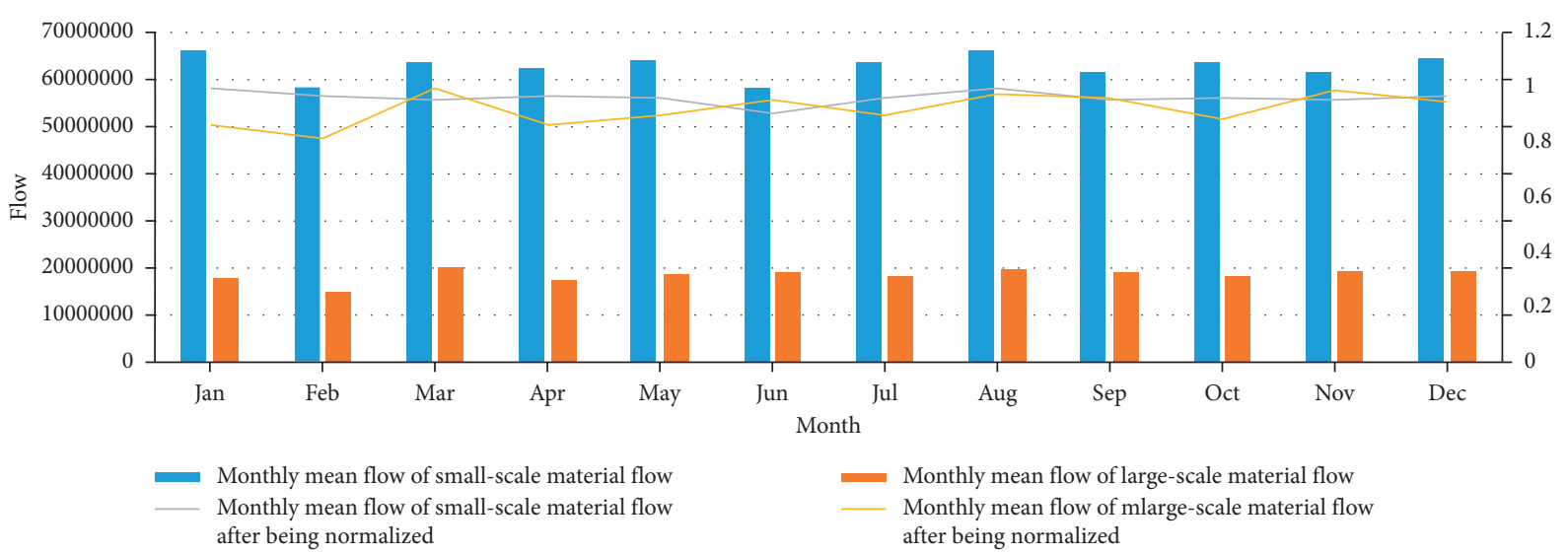

FIGURE 11: Monthly change trends of the monthly mean flows of the two kinds of material flows.

time to buy shoes or clothes in January, February, July, and August. The transportation of these goods is more suitable for small-scale flows. (b) March, April, and September are the offseasons for shopping and the maximum discount for goods may be $20 \%$. October to December is the peak of the discount season, and most businesses can offer a discount like "give 100 for every 1000." During this period, people's willingness to buy all kinds of goods (especially large commodities) is significantly higher than that of other months. Buying these large commodities will naturally result in a larger large-scale material flow. In general, the monthly mean flow change trends of the large-scale material flow and small-scale material flow are different. The varying amplitude of the small-scale material flow is relatively small. The contribution of the small-scale material flow to the total material flow is greater than that of the large-scale material flow.

3.3.2. Differences in the Daily Characteristics of the Two Kinds of Material Flows. The daily change trends of the mean flows of the two kinds of material flows are shown in Figure 12.

Figure 12 reveals the following. (1) From Monday to Friday, the large-scale material flow shows an upward trend, and the growth rate from Monday to Tuesday is obvious. Thursday is its most active period. (2) The small-scale material flow shows a slight downward trend from Monday to Wednesday with a significant increase from Thursday to Friday. (3) The peaks of the two kinds of material flows are staggered. (4) On weekends, the small-scale material flow and the large-scale material flow are significantly less than those on weekdays. Especially, the large-scale material flow is rapidly declining on weekends.

\subsubsection{Differences in the Hourly Characteristics of the Two} Kinds of Material Flows. The hourly change trends of the two kinds of material flows are shown in Figure 13.

(1) The large-scale material flow has three characteristic peaks with peak points at 6:00, 10:00-11:00, and 14:00. (2) The small-scale material flow shows the characteristic of double peaks. The peak points appear at 9:00-10:00 and 16: 00-17:00, which are staggered with the peaks of the largescale material flow. (3) On the whole, 6:00-17:00 is the active period for the two kinds of material flows and 00:00-5:00 and 18:00-23:00 are the relatively silent periods for material flows over the course of a whole day.

\subsection{Analysis of the Differences in the Spatial Characteristics.} In this section, we computed and found the top $10 \mathrm{road}$ sections for each kind of flow. Then, the spatial distribution characteristics of those top 10 road sections are analyzed. 


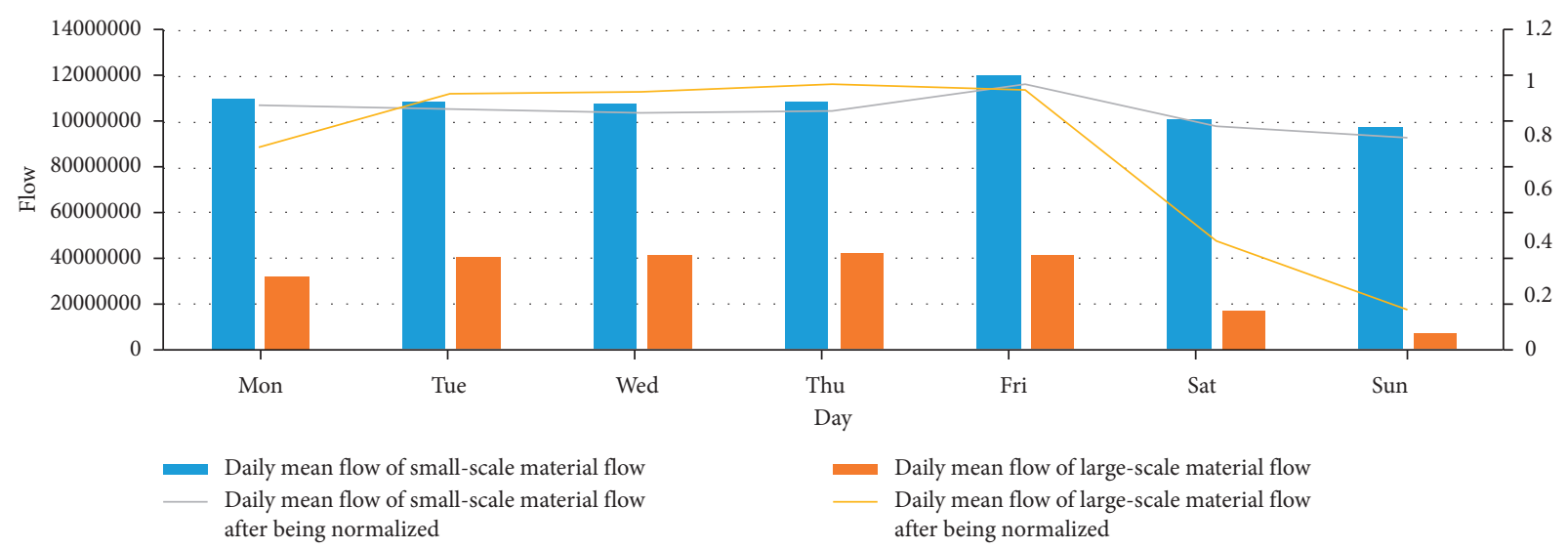

FIgURE 12: Daily change trends of daily mean flows of the two kinds of material flows.

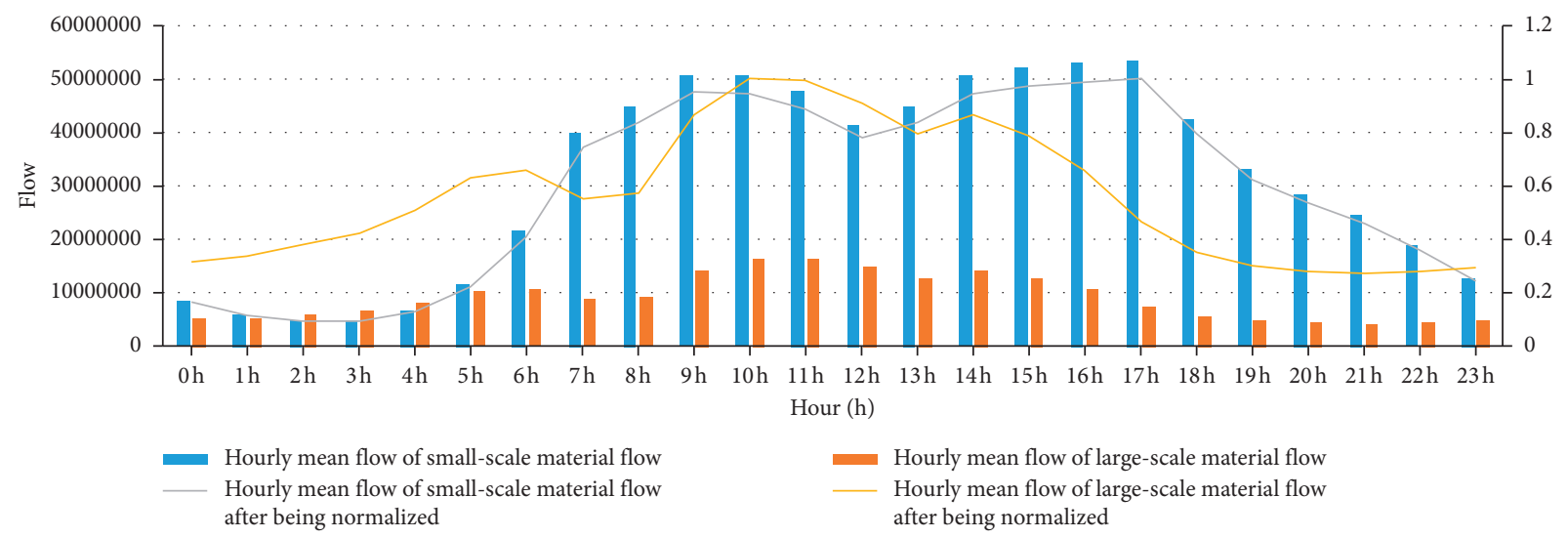

Figure 13: Hourly change trends of the hourly mean flows of the two kinds of material flows.

3.4.1. Analysis of the Differences among the Top 10 Road Sections. According to the monthly mean flow of each road section in different kinds of flows (such as the material flow, population flow, large-scale material flow, and small-scale material flow), we ordered all the road sections by their flows from high to low. Then, we selected the road sections which rank in the top 10 in different kinds of flows, respectively. The top 10 road sections are shown in Figures 14(a)-14(f).

We can find the following from Figure 14. (1) There are significant differences between the hot road sections of the minority population flow and the mass population flow, and only one road section is the same in top 10. (2) There are also obvious differences between the hot road sections of the small-scale material flow and the large-scale material flow, and only two of the top 10 sections are the same. (3) Although the top 10 rankings of the total population flow and total material are different, six of them are hot road sections of both the population flow and material flow.

3.4.2. Analysis of the Differences among County and City Units. Furthermore, this paper takes the administrative region (city or county) of each detection site as a unit to explore the differences in the population flow and logistics in each county or city. First, we calculate the outflow, inflow, and total flow (sum of the inflow and outflow) of the population flow and material flow in each city or county, respectively (Tables 3 and 4). Then, the natural breakpoint classification method [37] is adopted to classify the total population flow and total material flow in each city or county. The classification results are shown in Figures 15(a) and 15(b).

The classification results in Figure 15 show the following. (1) For a single city or county, the inflow and outflow of the population flow and material flow are relatively balanced. (2) In terms of the total population flow and total material flow, there are obvious grade differences among counties and cities, which are as follows. (a) New Taipei City, Taoyuan City, Taichung City, and Tainan City are the core areas of the TToF while Taipei City, Changhua County, and Kaohsiung City are the subcore areas. (b) Hsinchu County, Miaoli County, and Yunlin County are located on the third level while Nantou County and Chiayi County are on the fourth level. The counties and cities on these two levels connect the core area and subcore area of the TToF and receive their outflows. (c) Due to its geographical disadvantages, Keelung City, Yilan County, and Pingtung County are on the lowest level. (3) From the ranking of the total flow, the rankings of counties and cities regarding their total population flow and material flow are different, but the differences are not obvious. The core areas of the population flow are also the core areas of the material flow. 


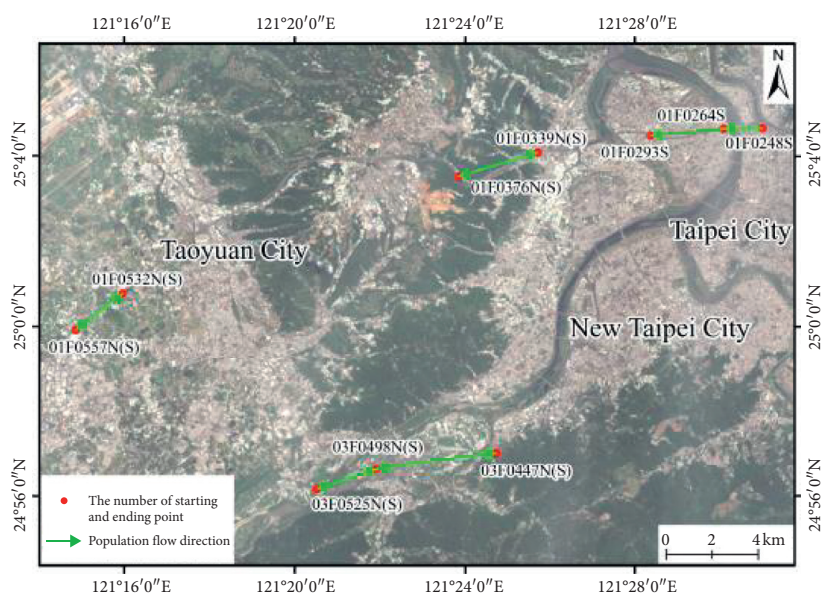

(a)

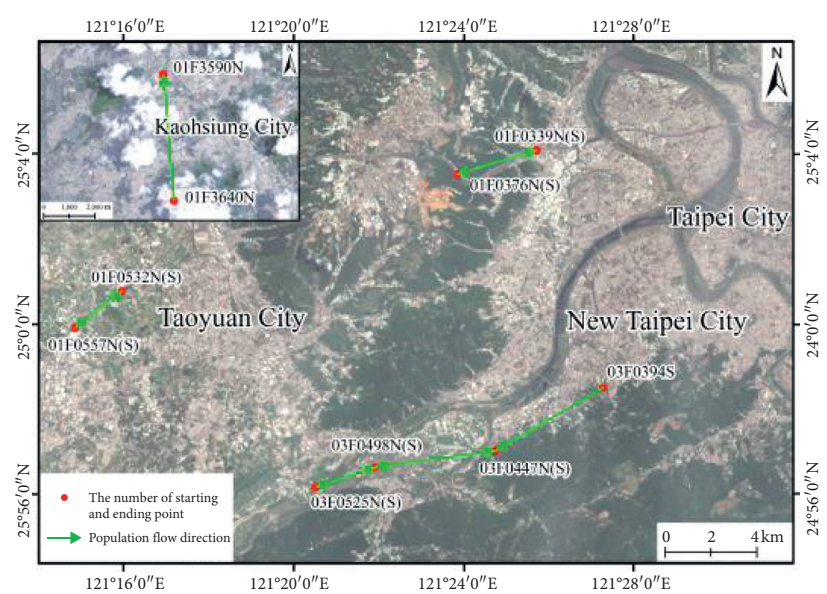

(c)

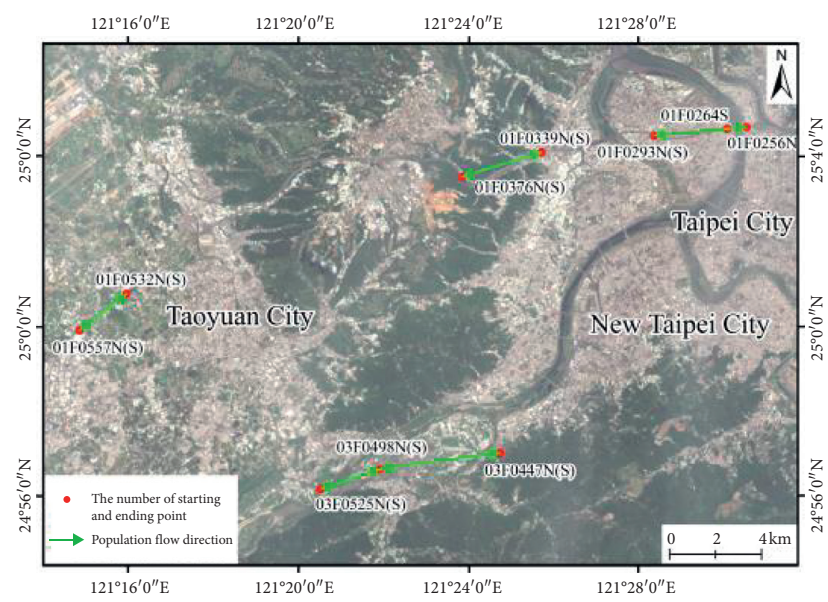

(e)

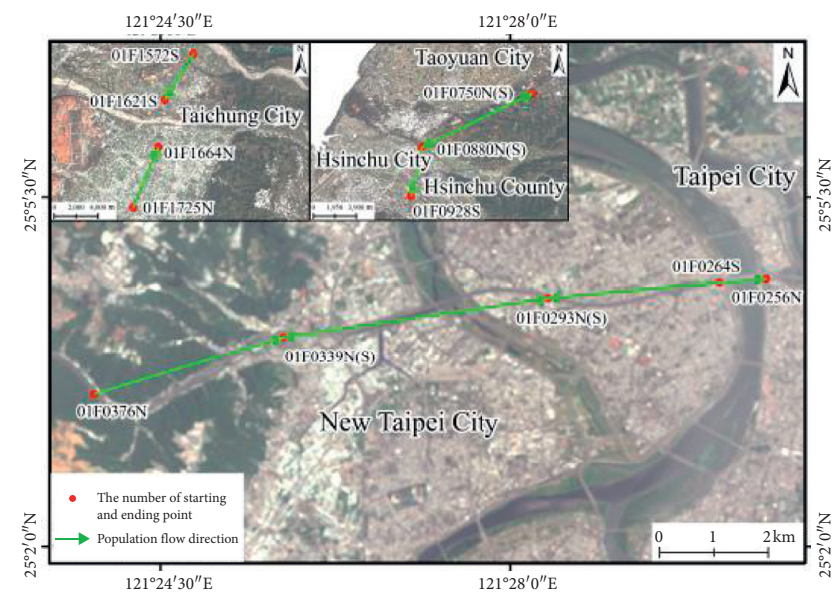

(b)

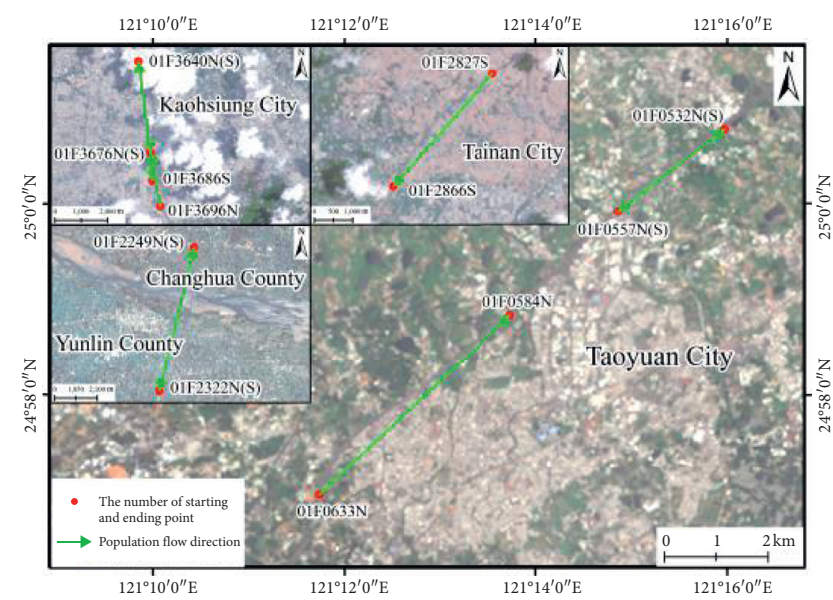

(d)

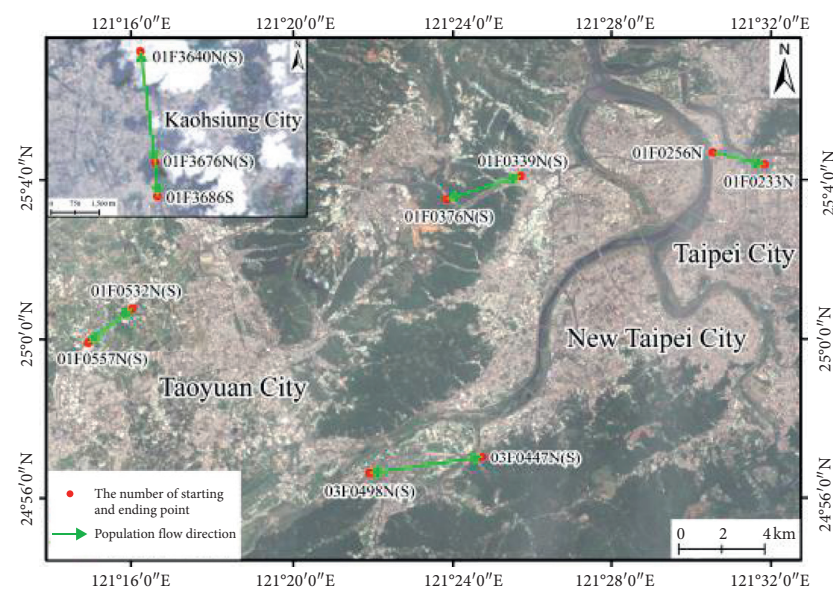

(f)

Figure 14: Top ten road sections with the monthly mean flow of each kind of flow. (a) Minority population flow. (b) Mass population flow. (c) Small-scale material flow. (d) Large-scale material flow. (e) Population flow. (f) Material flow.

3.4.3. Analysis of the Influence Factors of the Intensity of the TToF. Here, we explore the possible factors affecting the intensity of the TToF in each county and city. Based on the availability of data, 23 indexes that may impact the intensity are preliminarily selected from "the statistical yearbook of Taiwan Province 2016" [38]. Then, adopting the method of literature [39], the correlation coefficient analysis and variation coefficient analysis are carried out for each index to delete the redundant indexes. Finally, 12 indexes are obtained that are used in the impact factor analysis. Finally, the statistical product and service solutions (SPSS) software is used to analyze the correlation between the total population 
Table 3: Statistics of the material flow in counties and cities.

\begin{tabular}{lccccc}
\hline Origin & $\begin{array}{c}\text { Annual } \\
\text { outflow }\end{array}$ & Destination & $\begin{array}{c}\text { Annual } \\
\text { inflow }\end{array}$ & $\begin{array}{c}\text { Sum of annual outflow and } \\
\text { inflow }\end{array}$ & $\begin{array}{c}\text { Ranking based on sum of annual outflow } \\
\text { and inflow }\end{array}$ \\
\hline Kaohsiung City & 91966723 & Kaohsiung City & 91996712 & 183963435 & 5 \\
Keelung City & 16798794 & Keelung City & 16998386 & 33797180 & 14 \\
Chiayi County & 36462400 & Chiayi County & 36623926 & 73086326 & 11 \\
Miaoli County & 60403723 & Miaoli County & 60660516 & 121064239 & 8 \\
Nantou County & 30185811 & Nantou County & 29885821 & 60071632 & 12 \\
Pingtung & 9188146 & Pingtung & 9143544 & 18331690 & 15 \\
County & 63888255 & Caipei City & 62984284 & 126872539 & 7 \\
Taipei City & 109586595 & Tainan City & 109564590 & 219151185 & 4 \\
Tainan City & 111740437 & Taichung City & 112128617 & 223869054 & 3 \\
Taichung City & 117372318 & Taoyuan City & 118157534 & 235529852 & 2 \\
Taoyuan City & 137515698 & New Taipei City & 137525403 & 275041101 & 1 \\
New Taipei City & 22262323 & Hsinchu City & 22173607 & 44435930 & 13 \\
Hsinchu City & 54000472 & Hsinchu County & 53926057 & 107926529 & 9 \\
Hsinchu & 8269761 & Yilan County & 8319961 & 16589722 & 16 \\
County & 43568792 & Yunlin County & 43605244 & 87174036 & 10 \\
Yilan County & Changhua & 63476452 & 127436858 & 6 \\
Yunlin County & Changhua & 63960406 & County & &
\end{tabular}

TABLE 4: Statistics of the population flow in counties and cities.

\begin{tabular}{|c|c|c|c|c|c|}
\hline Origin & $\begin{array}{l}\text { Annual } \\
\text { outflow }\end{array}$ & Destination & $\begin{array}{l}\text { Annual } \\
\text { inflow }\end{array}$ & $\begin{array}{l}\text { Sum of annual outflow and } \\
\text { inflow }\end{array}$ & $\begin{array}{c}\text { Ranking based on sum of annual outflow } \\
\text { and inflow }\end{array}$ \\
\hline Kaohsiung City & 215220039 & Kaohsiung City & 214982233 & 430202272 & 5 \\
\hline Keelung City & 55542552 & Keelung City & 55780534 & 111323086 & 14 \\
\hline Chiayi County & 91870913 & Chiayi County & 92038329 & 183909242 & 12 \\
\hline Miaoli County & 169542618 & Miaoli County & 170424104 & 339966722 & 7 \\
\hline Nantou County & 101702641 & Nantou County & 100944052 & 202646693 & 11 \\
\hline $\begin{array}{l}\text { Pingtung } \\
\text { County }\end{array}$ & 27436449 & $\begin{array}{l}\text { Pingtung } \\
\text { County }\end{array}$ & 27451774 & 54888223 & 15 \\
\hline Taipei City & 176428753 & Taipei City & 174052818 & 350481571 & 6 \\
\hline Tainan City & 273274745 & Tainan City & 273504078 & 546778823 & 4 \\
\hline Taichung City & 323937927 & Taichung City & 325030196 & 648968123 & 3 \\
\hline Taoyuan City & 326718417 & Taoyuan City & 328732869 & 655451286 & 2 \\
\hline New Taipei City & 437187131 & New Taipei City & 437032237 & 874219368 & 1 \\
\hline Hsinchu City & 61698489 & Hsinchu City & 61251123 & 122949612 & 13 \\
\hline $\begin{array}{l}\text { Hsinchu } \\
\text { County }\end{array}$ & 163558445 & Hsinchu County & 164058190 & 327616635 & 8 \\
\hline Yilan County & 26384160 & Yilan County & 26534079 & 52918239 & 16 \\
\hline Yunlin County & 111735551 & Yunlin County & 111722029 & 223457580 & 10 \\
\hline $\begin{array}{l}\text { Changhua } \\
\text { County }\end{array}$ & 161392150 & $\begin{array}{l}\text { Changhua } \\
\text { County }\end{array}$ & 160092335 & 321484485 & 9 \\
\hline
\end{tabular}

flow, the total material flow, and the relevant index data of each county and city. The correlation matrices we get are shown in Tables 5 and 6.

The following can be seen from the correlation matrix in Tables 5 and 6. (1) In terms of the population flow, we get the following. (a) The factors related to the intensity of the population flow in order are the NPBSI (0.897), NFIO (0.895), SSC (0.864), EMC (0.861), TPC (0.858), HHC (0.836), NPBTI (0.690), and BSC (0.687). (b) Among these factors, the NFIO and EMC ranked second and fourth, respectively, which indicates that the main reason for the population flow of Taiwan's expressways is due to work needs. (c) The correlation between the intensity of the population flow and the SSC is significantly higher than that with the BSC. This confirms that the main composition of Taiwan's expressway population flow is the minority population flow. (2) In terms of the material flow, we can get the following. (a) The factors related to the material flow's intensity in order are the NFIO (0.875), NPBSI (0.867), EMC (0.858), TPC (0.854), HHC (0.831), STC (0.814), BTC (0.801), and NPBTI (0.693). (b) The top 4 factors most relevant to the intensity of the material flow are the NFIO, EMC, NPBSI, and TPC. This shows that the intensity of the material flow is greatly influenced by the population size, especially the number of employed people. In addition, it also proves the reasonability of the intensity classification 


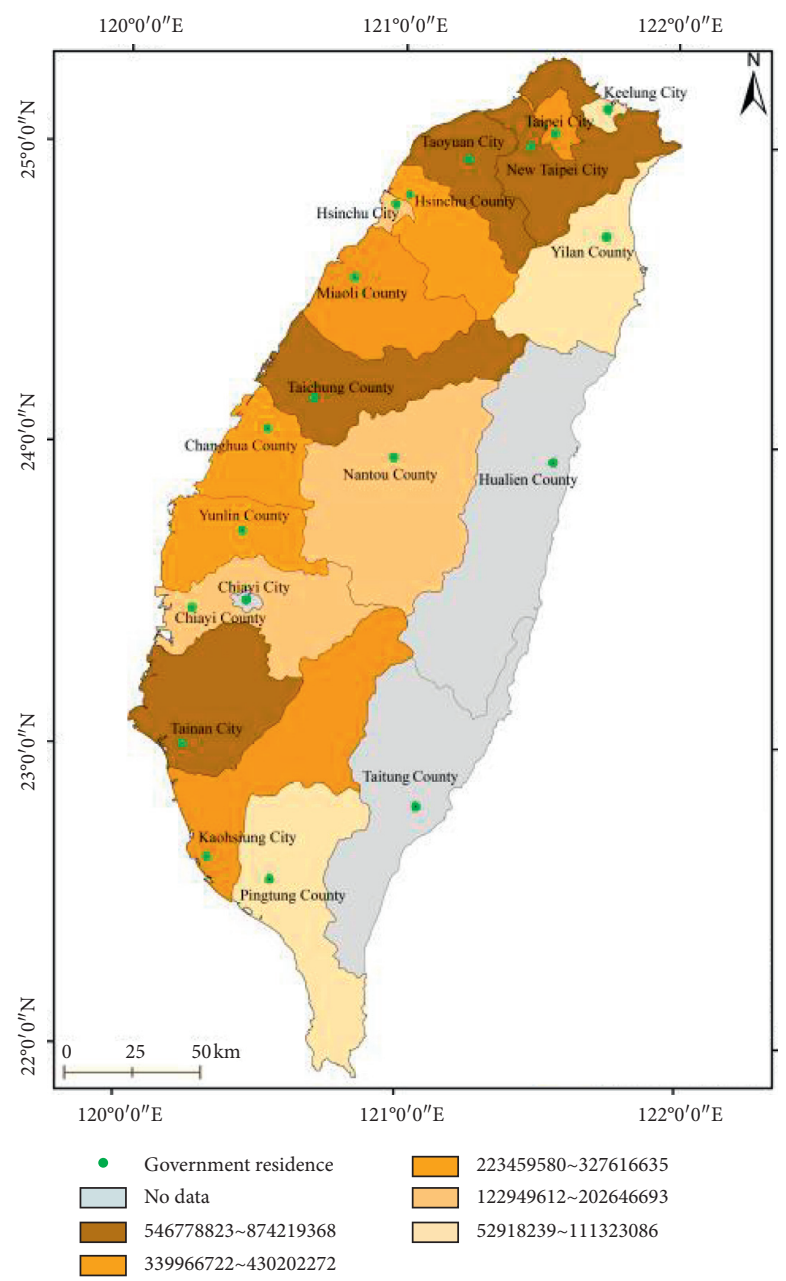

(a)

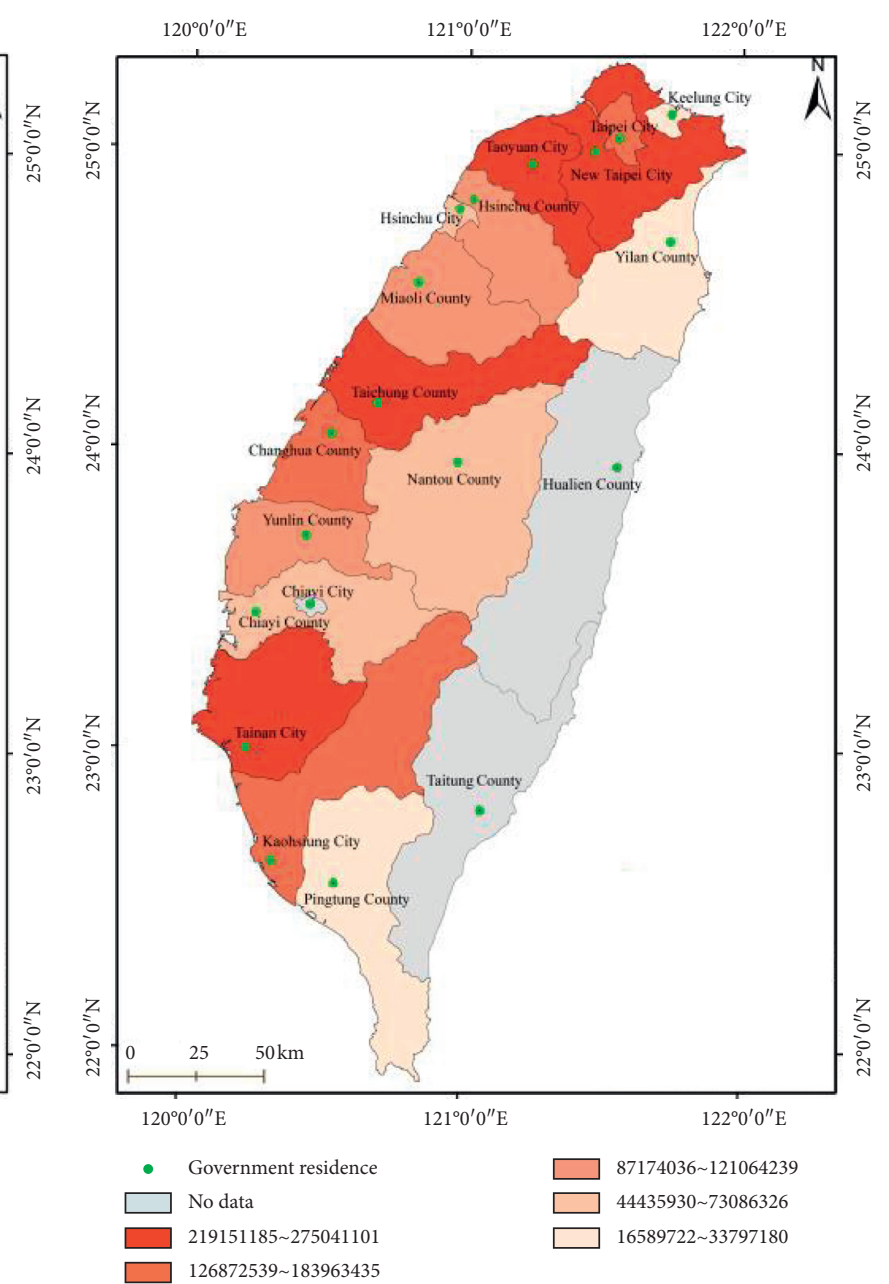

(b)

Figure 15: Classification of the counties and cities based on the total circulation. (a) Classification of the population flow. (b) Classification of the material flow.

Table 5: Analysis of the influencing factors of the population flow's intensity.

\begin{tabular}{lccccccccccccc}
\hline & TPFC & TPC & HHC & HHVC & PD & EMC & FCPR & NFIO & BSC & SSC & NPBPI & NPBSI & NPBTI \\
\hline TPFC & 1 & $0.858^{* *}$ & $0.836^{* *}$ & -0.057 & 0.055 & $0.861^{* *}$ & 0.053 & $0.895^{* *}$ & $0.687^{* *}$ & $0.864^{* *}$ & 0.139 & $0.897^{* *}$ & $0.690^{* *}$ \\
TPC & $0.858^{* *}$ & 1 & $0.996^{* *}$ & -0.262 & 0.326 & $0.999^{* *}$ & -0.232 & $0.814^{* *}$ & $0.915^{* *}$ & $0.975^{* *}$ & 0.395 & $0.932^{* *}$ & $0.926^{* *}$ \\
HHC & $0.836^{* *}$ & $0.996^{* *}$ & 1 & -0.330 & 0.354 & $0.996^{* *}$ & -0.273 & $0.772^{* *}$ & $0.931^{* *}$ & $0.957^{* *}$ & 0.417 & $0.908^{* *}$ & $0.931^{* *}$ \\
HHVC & -0.057 & -0.262 & -0.330 & 1 & -0.401 & -0.258 & $0.575^{*}$ & 0.079 & -0.397 & -0.166 & -0.215 & -0.085 & -0.339 \\
PD & 0.055 & 0.326 & 0.354 & -0.401 & 1 & 0.316 & -0.427 & -0.089 & $0.632^{* *}$ & 0.298 & -0.131 & 0.133 & $0.609^{*}$ \\
EMC & $0.861^{* *}$ & $0.999^{* *}$ & $0.996^{* *}$ & -0.258 & 0.316 & 1 & -0.240 & $0.818^{* *}$ & $0.913^{* *}$ & $0.970^{* *}$ & 0.387 & $0.934^{* *}$ & $0.920^{* *}$ \\
FCPR & 0.053 & -0.232 & -0.273 & $0.575^{*}$ & -0.427 & -0.240 & 1 & 0.030 & -0.390 & -0.113 & -0.015 & -0.127 & -0.319 \\
NFIO & $0.895^{* *}$ & $0.814^{* *}$ & $0.772^{* *}$ & 0.079 & -0.089 & $0.818^{* *}$ & 0.030 & 1 & $0.583^{*}$ & $0.835^{* *}$ & 0.182 & $0.954^{* *}$ & $0.592^{*}$ \\
BSC & $0.687^{* *}$ & $0.915^{* *}$ & $0.931^{* *}$ & -0.397 & $0.632^{* *}$ & $0.913^{* *}$ & -0.390 & $0.583^{*}$ & 1 & $0.856^{* *}$ & 0.248 & $0.791^{* *}$ & $0.969^{* *}$ \\
SSC & $0.864^{* *}$ & $0.975^{* *}$ & $0.957^{* *}$ & -0.166 & 0.298 & $0.970^{* *}$ & -0.113 & $0.835^{* *}$ & $0.856^{* *}$ & 1 & 0.383 & $0.922^{* *}$ & $0.914^{* *}$ \\
NPBPI & 0.139 & 0.395 & 0.417 & -0.215 & -0.131 & 0.387 & -0.015 & 0.182 & 0.248 & 0.383 & 1 & 0.254 & 0.314 \\
NPBSI & $0.897^{* *}$ & $0.932^{* *}$ & $0.908^{* *}$ & -0.085 & 0.133 & $0.934^{* *}$ & -0.127 & $0.954^{* *}$ & $0.791^{* *}$ & $0.922^{* *}$ & 0.254 & 1 & $0.782^{* *}$ \\
NPBTI & $0.690^{* *}$ & $0.926^{* *}$ & $0.931^{* *}$ & -0.339 & $0.609^{*}$ & $0.920^{* *}$ & -0.319 & $0.592^{*}$ & $0.969^{* *}$ & $0.914^{* *}$ & 0.314 & $0.782^{* *}$ & 1 \\
\hline
\end{tabular}

result for the TToF. (c) The correlation between the intensity of the material flow and STC is slightly higher than that with the BTC. It confirms that the proportion of the small-scale material flow with respect to the total material flow is higher than that of the large-scale material flow. (3) From a comprehensive point of view, different industries and structures will also have great impacts on the intensity of the TToF, which is shown by the correlations between the 
TABLE 6: Analysis of the influencing factors of the material flow's intensity.

\begin{tabular}{lccccccccccccc}
\hline & TMFC & TPC & HHC & HHVC & PD & EMC & FCPR & NFIO & BTC & STC & NPBPI & NPBSI & NPBTI \\
\hline TMFC & 1 & $0.854^{* *}$ & $0.831^{* *}$ & -0.028 & 0.047 & $0.858^{* *}$ & 0.084 & $0.875^{* *}$ & $0.801^{* *}$ & $0.814^{* *}$ & 0.226 & $0.867^{* *}$ & $0.693^{* *}$ \\
TPC & $0.854^{* *}$ & 1 & $0.996^{* *}$ & -0.262 & 0.326 & $0.999^{* *}$ & -0.232 & $0.814^{* *}$ & $0.780^{* *}$ & $0.864^{* *}$ & 0.395 & $0.932^{* *}$ & $0.926^{* *}$ \\
HHC & $0.831^{* *}$ & $0.996^{* *}$ & 1 & -0.330 & 0.354 & $0.996^{* *}$ & -0.273 & $0.772^{* *}$ & $0.748^{* *}$ & $0.828^{* *}$ & 0.417 & $0.908^{* *}$ & $0.931^{* *}$ \\
HHVC & -0.028 & -0.262 & -0.330 & 1 & -0.401 & -0.258 & $0.575^{*}$ & 0.079 & 0.031 & 0.088 & -0.215 & -0.085 & -0.339 \\
PD & 0.047 & 0.326 & 0.354 & -0.401 & 1 & 0.316 & -0.427 & -0.089 & -0.064 & 0.016 & -0.131 & 0.133 & $0.609^{*}$ \\
EMC & $0.858^{* *}$ & $0.999^{* *}$ & $0.996^{* *}$ & -0.258 & 0.316 & 1 & -0.240 & $0.818^{* *}$ & $0.771^{* *}$ & $0.864^{* *}$ & 0.387 & $0.934^{* *}$ & $0.920^{* *}$ \\
FCPR & 0.084 & -0.232 & -0.273 & $0.575^{*}$ & -0.427 & -0.240 & 1 & 0.030 & 0.116 & -0.013 & -0.015 & -0.127 & -0.319 \\
NFIO & $0.875^{* *}$ & $0.814^{* *}$ & $0.772^{* *}$ & 0.079 & -0.089 & $0.818^{* *}$ & 0.030 & 1 & $0.777^{* *}$ & $0.840^{* *}$ & 0.182 & $0.954^{* *}$ & $0.592^{*}$ \\
BTC & $0.801^{* *}$ & $0.780^{* *}$ & $0.748^{* *}$ & 0.031 & -0.064 & $0.771^{* *}$ & 0.116 & $0.777^{* *}$ & 1 & $0.909^{* *}$ & $0.614^{*}$ & $0.760^{* *}$ & $0.628^{* *}$ \\
STC & $0.814^{* *}$ & $0.864^{* *}$ & $0.828^{* *}$ & 0.088 & 0.016 & $0.864^{* *}$ & -0.013 & $0.840^{* *}$ & $0.909^{* *}$ & 1 & 0.448 & $0.858^{* *}$ & $0.742^{* *}$ \\
NPBPI & 0.226 & 0.395 & 0.417 & -0.215 & -0.131 & 0.387 & -0.015 & 0.182 & $0.614^{*}$ & 0.448 & 1 & 0.254 & 0.314 \\
NPBSI & $0.867^{* *}$ & $0.932^{* *}$ & $0.908^{* *}$ & -0.085 & 0.133 & $0.934^{* *}$ & -0.127 & $0.954^{* *}$ & $0.760^{* *}$ & $0.858^{* *}$ & 0.254 & 1 & $0.782^{* *}$ \\
NPBTI & $0.693^{* *}$ & $0.926^{* *}$ & $0.931^{* *}$ & -0.339 & $0.609^{*}$ & $0.920^{* *}$ & -0.319 & $0.592^{*}$ & $0.628^{* *}$ & $0.742^{* *}$ & 0.314 & $0.782^{* *}$ & 1 \\
\hline
\end{tabular}

Note. TPC, HHC, HHVC, PD, EMC, FCPR, NFIO, NPBPI, NPBSI, and NPBTI in Tables 5 and 6 represent total population (thousand people), number of households (thousand households), number of households (person/household), population density (person/ $\mathrm{km}^{2}$ ), number of employees (thousand people), household car penetration rate, number of factories in operation (no.), and number of profit-making enterprises in the first industry and second industry (no.), respectively; TPFC, BSC, and SSC in Table 5 represent the total flow of population, subtotal of buses (vehicles), and subtotal of buses (vehicles). TMFC, BTC, and STC in Table 6 represent the total flow of material, subtotal of trucks (vehicles), and subtotal of trucks (vehicles), respectively. ${ }^{* *}$ When the confidence (double test) is 0.01 , the correlation is significant. ${ }^{*}$ When the confidence (double test) is 0.05 , the correlation is significant. The first row of data of each index is Pearson correlation, and the second row is significance (double tail). Sample capacity $n$ is 16 .

intensity of the TToF and the NPBPI, NPBSI and NPBTI being significantly different. The contribution of the second industry to the TToF is greater than that of the third industry, and it is further greater than that of the first industry.

3.5. Spatial and Temporal Autocorrelation Analysis. To further explore the spatial-temporal correlation of Taiwan's expressway network, ST-ACF and CCF are adopted to analyze the global spatial-temporal autocorrelation and local spatial-temporal autocorrelation, respectively.

3.5.1. Global Spatial-Temporal Autocorrelation Analysis. In this paper, the spatial first-order adjacency matrix and the second-order adjacency matrix of each road section are used as the spatial weight matrix to calculate the ST-ACF index of all road sections. Meanwhile, to better reflect the temporal and spatial differences in the spatial-temporal correlation of Taiwan's expressway network, we analyze the changes of STACF index in the early peak (8:00-11:00), the middle peak (11:00-16:00), and the late peak (16:00-18:00) periods. The calculation results of the ST-ACF index are shown in Figure 16.

The following can be seen from Figure 16. (1) The STACF value changes periodically over the whole day (Figure 16(d)), and the change period is consistent with the time length of the analysis (every 5 minutes, 288 intervals in the whole day). (2) The spatial-temporal correlation is negatively correlated with the spatial distance, which is reflected by the ST-ACF value in Figure 16(d) decreasing as the spatial order increasing. (a) When the spatial order is first order, the ST-ACF value is greater than 0 and the degree of confidence is greater than $95 \%$, showing a significant positive correlation. (b) The spatial-temporal correlation is not significant when the spatial order is second order. This shows that the spatial-temporal correlation of Taiwan's expressway network is mainly reflected in the direct adjacent road sections, but not in the indirect connected road sections. (3) Compared with the early peak and the late peak, the ST-ACF value in the middle peak is significantly higher, and it changes faster. As a whole, the different traffic patterns in different periods for Taiwan's expressway network show that the spatial-temporal autocorrelation has the characteristics of time dynamics and spatial heterogeneity.

\subsubsection{Local Spatial-Temporal Autocorrelation Analysis.} The global spatial-temporal autocorrelation analysis describes Taiwan's expressway network as a whole, but it may cover up some local heterogeneity. Therefore, it is necessary to analyze the local spatial-temporal correlation. Considering the fact that the core areas of the TToF are located in the northern part of Taiwan Province and considering the ranking of the intensity of the TToF in each detection section, five consecutive and intersecting road sections (as shown in Figure 17(a)) are selected from the top 50 sections from the northern part of Taiwan Province for the local spatial-temporal autocorrelation analysis. Then, using the method in literature [36], the CCF values of different periods (early peak, middle peak, and late peak) in the spatial firstorder adjacency matrix are calculated. The results are shown in Figure 17.

(1) The CCF values in different road sections show obvious heterogeneity. (a) In the middle peak, with the spatial first-order adjacency matrix and time delay $s$ being zero, the CCF values of the five road sections vary from -0.2 to 0.35 . This means that there are significant differences in the correlation between some road sections and their spatial first-order adjacent road sections. (b) As shown in Figure 17(b), road sections 1,2 , and 3 show a strong positive correlation (CCF values are greater than zero). Road section 4 has a CCF close to zero, which means that it has a weak correlation with its directly adjacent road 


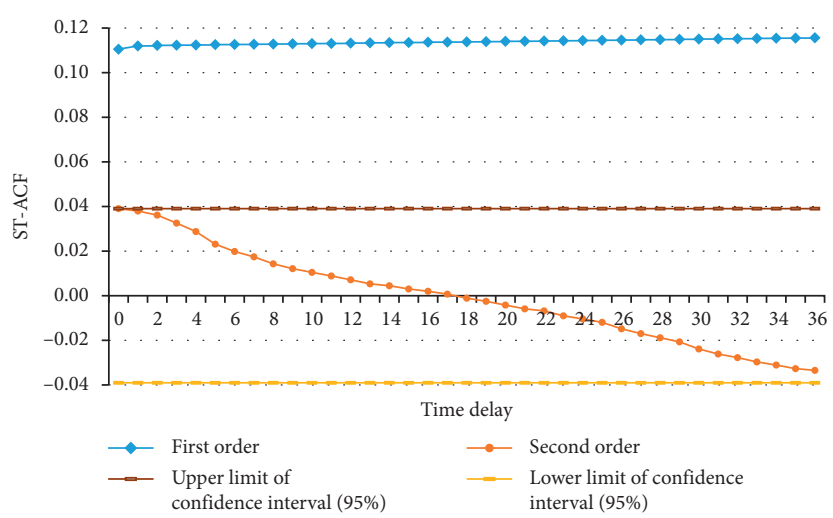

(a)

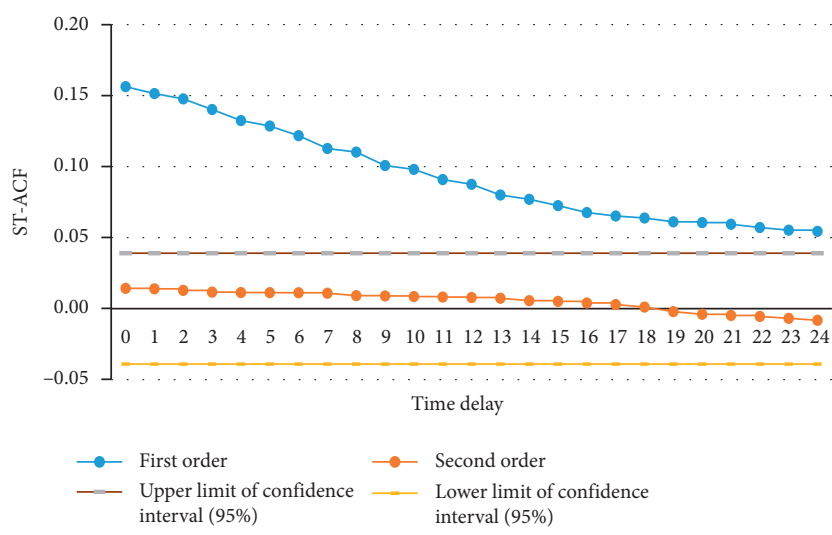

(c)

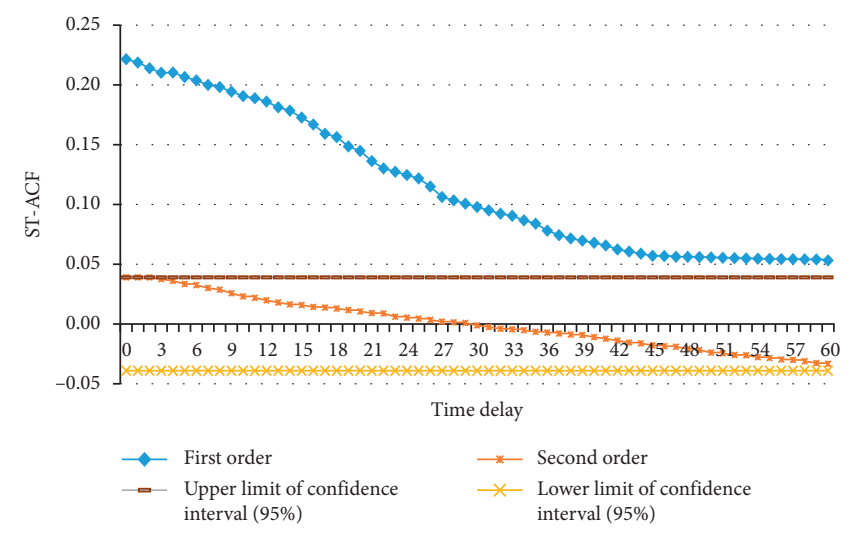

(b)

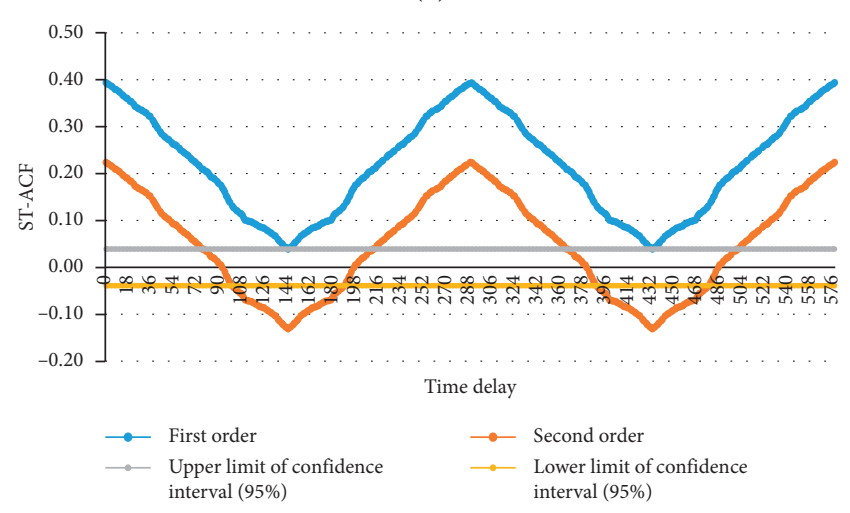

(d)

Figure 16: ST-ACF value analysis of Taiwan's expressway network. (a) Early peak. (b) Middle peak. (c) Late peak. (d) All day long.

sections. Meanwhile, road section 5 shows a negative correlation. (c) The correlation of each road section varies with time. For example, in the late peak period, the correlation of road sections 3 and 5 gradually changes from negative to positive, the correlation of road sections 1 and 2 changes from positive to negative, and section 4 does not change much from beginning to end.

(2) The correlation of the same road section also changes in different periods. For example, the correlation of section 3 shows a positive correlation in the early peak period and a negative correlation in the middle and late peak periods. It shows that the autocorrelation structure also changes with time.

\section{Conclusion}

The population flow and material flow are fundamental elements of space of flows. The main shortcoming of most of the existing works related to TToF is that they ignore the differences of spatiotemporal characteristics in different scales and the correlation between them. To grasp the spatial-temporal differences of TToF in specific research area deeply and accurately, more detailed work is needed. In this paper, we study the spatial-temporal characteristics of the population flow and material flow in Taiwan Province and analyze the spatial-temporal autocorrelation of Taiwan's expressway network based on the ETC data of Taiwan Province in 2017. The population flow and material flow are distinguished by vehicle types in the experimental data. To fully explore the potential value of the data, we adopt a multimembership and layer-by-layer refinement method to carry out this research to discover Taiwanese travel habits and the material flow patterns of Taiwan Province. The thoughts of multimembership and layer-by-layer refinement are mainly embodied in four aspects. (1) The population flow is divided into two subtypes named the "minority population flow" and the "mass population flow," while the material flow is divided into the "small-scale material flow" and the "large-scale material flow" correspondingly. (2) The differences among the time characteristics of these kinds of flows are studied in monthly, daily, and hourly units, respectively. (3) The spatial differences of the different types of flows are studied under different scales (road sections, cities, and counties). (4) The ST-ACF and CCF are adopted to study the global and local spatial-temporal autocorrelation of Taiwan's expressway network, respectively. The results show the following. (1) Both the different temporal characteristics and the spatial characteristics of the TToF in Taiwan Province are obvious. (2) The flow intensity of the TToF is largely influenced by some objective factors, such as the population size (especially the number of employed 


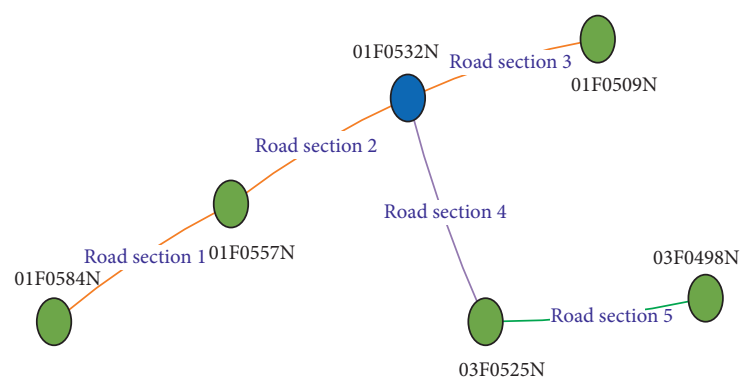

(a)

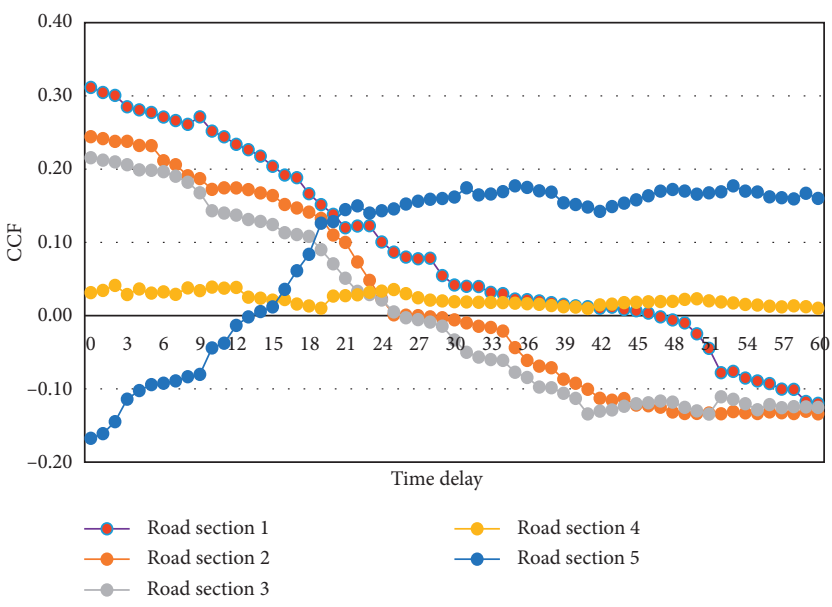

(c)

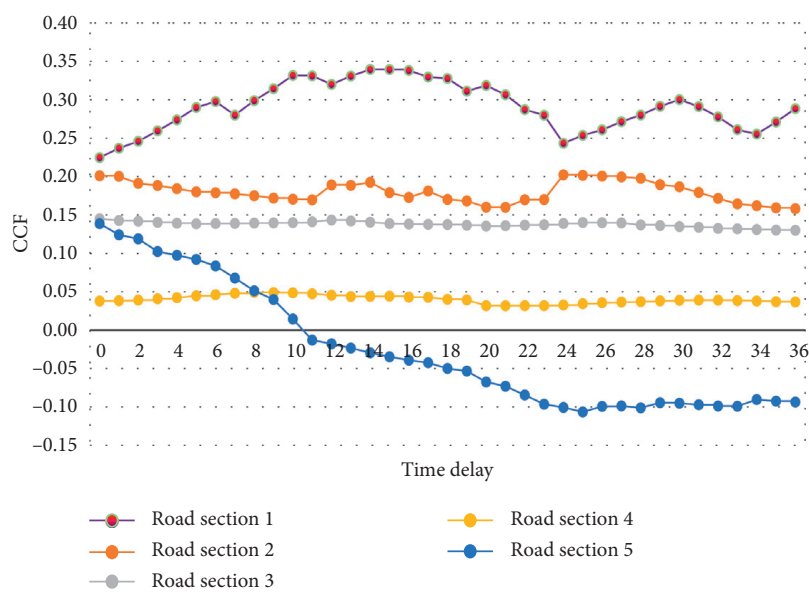

(b)

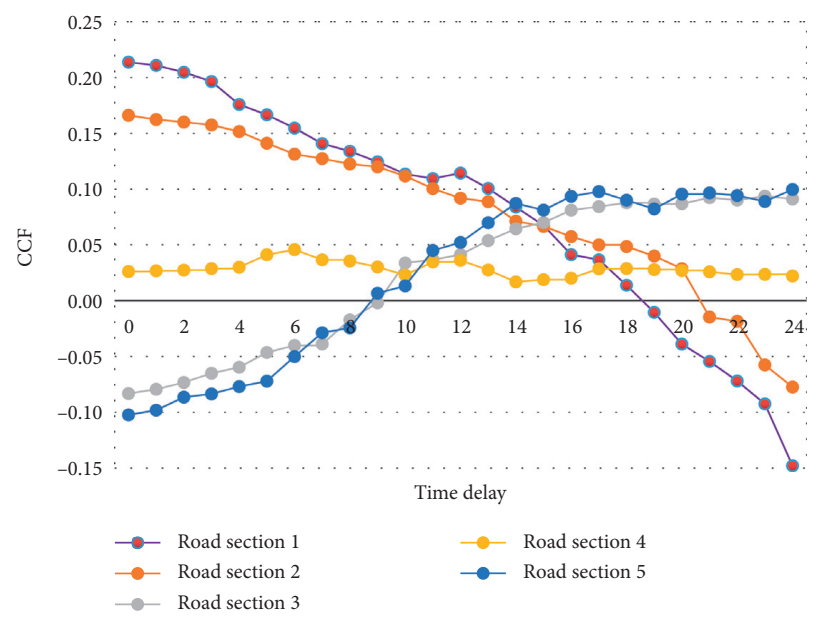

(d)

FIGURE 17: Road section selection and the CCF value changes of each section in different periods. (a) Five selected road sections. (b) Early peak. (c) Middle peak. (d) Late peak.

population), industrial structure, and so on. (3) There is a significant positive spatial-temporal correlation in Taiwan's expressway network, but this correlation is mainly reflected in the spatial first-order adjacent road sections.

The value of our work is mainly embodied in four aspects: (1) constructing a research framework for studying the TToF by adopting the multimembership and layer-by-layer refinement analysis idea, (2) helping decision makers and some other researchers to further understand Taiwanese's travel habits and the material flow patterns of Taiwan Province, (3) providing some basis for decision makers to conduct traffic planning and adjust industrial layout, and (4) showing another novel use of the ETC data. However, there are also some limitations in this paper. (1) The annual gross domestic product (GDP) of tourism is relatively considerable in Taiwan Province, and so the minority flow generated by the tourism industry will inevitably affect the total population flow of Taiwan Province. However, limited by the availability of data, factors such as the number of tourists in each county and city and their tourism incomes are not taken into account in the process of the factor analysis of the intensity of the TToF. (2) The current work of this paper mainly focuses on the analysis of the flow "intensity" of each type of flow and fails to properly excavate the value of the flow "direction." In the follow-up work, the starting sites and ending sites and their administrative regions could be used to construct the population flow and material flow correlated network of site-to-site and county (city)-tocounty (city) networks. Then, the social network analysis method could be used to evaluate the whole network and each node in the network.

\section{Data Availability}

Our data have been uploaded to Dryad and assigned with a DOI. The data are approved by Dryad curators, and the following temporary link can be used for obtaining the data: https://datadryad.org/stash/share/OhiOnewzuI59cxMXgR WEIma4TqS_05hUulUQGRlOCkQ. The data can also be downloaded from the following website: http://tisvcloud. freeway.gov.tw/history/TDCS/M05A/. 


\section{Conflicts of Interest}

The authors declare that they have no conflicts of interest.

\section{Acknowledgments}

We would like to thank all the partners who took part in the study. This research was funded by the National Natural Science Foundation of China (grant no. 61872086), the startup project of doctoral research of Fujian Normal University, and the project of Fujian Provincial Department of Education (grant no. JAT170140).

\section{References}

[1] D. Xiaomin and C. Jianbao, "Empirical analysis on impacts of migration with and without Hukou change on China's provincial economy," Population Research, vol. 34, no. 3, pp. 77-88, 2010.

[2] Y. Wenjie, S. Jianfu, L. Huanhuan et al., "Quantitative relationship between economic development and population migration in Germany from 1999 to 2009," Commercial Times, no. 3, pp. 15-16, 2011.

[3] L. Xiaoyang, L. Tianzhu, and Z. Qi, "Interaction study between population mobility and economic growth: evidence from Chongqing," Chinese Journal of Population Science, vol. 6, pp. 46-55, 2015.

[4] W. Zhiming and Z. Lun, "The interpretation of Fei HsiaoTung and Ebenezer Howard's thoughts on migration flows and urbanization," Urban Studies, vol. 17, no. 9, pp. 13-19, 2010.

[5] Z. Min, C. Chen, and Y. Haiwen, "On urbanization driven by rural-urban migration and its policy implications," Urban Planning Forum, vol. 2, pp. 1-9, 2013.

[6] Y. Yonghua, The effect on the urbanization process by population flow: theoretical analysis and empirical study, Ph.D. dissertation, Lanzhou University, Lanzhou, China, 2016.

[7] L. Yang, L. Hui, and T. Qiang, "Spatial-temporal patterns of China's interprovincial migration during 1985-2010," Geographical Research, vol. 34, no. 6, pp. 1135-1148, 2015.

[8] W. Jing, L. Benteng, and L. Yurui, "Spatial-temporal characteristics and influencing factors of population distribution and floating changes in Beijing-Tianjin-Hebei region," Geographical Research, vol. 37, no. 9, pp. 1802-1817, 2018.

[9] M. Zhifei, Y. Shanggang, Z. Yu et al., "Spatial distribution, flowing rules and forming mechanism of inter-cities floating population in China," Geographical Research, vol. 38, no. 4, pp. 926-936, 2019.

[10] P. Jinghu and L. Jianbo, "Research on spatial pattern of population mobility among cities: a case study of "Tencent migration" big data in "national day-mid-autumn festival" vacation," Geographical Research, vol. 38, no. 7, pp. 1678-1693, 2019.

[11] H. Xiaolan and X. Kefeng, "Discussion on the integration and optimization of logistics, human flow, information flow and capital flow," Social Sciences in Yunnan, vol. 6, pp. 68-72, 2016.

[12] Z. Yaojun and C. Qiao, "Spatial patterns of population mobility and determinants of inter-provincial migration in China," Population Research, vol. 38, no. 5, pp. 54-71, 2014.

[13] C. Xiushan and Z. Keyun, Regional Economic Theory, The Commercial Press, Beijing, China, 2010.
[14] H. Yong and L. Congjiu, "Research on the logistics mode of Chinese enterprises," Logistics Technology, vol. 3, pp. 1-3, 2006.

[15] Q. Huimin and H. Jiang, "An empirical study on the selection of B2C cross-border e-commerce logistics model," Commercial Research, vol. 62, no. 12, pp. 118-125, 2016.

[16] X. Jie, Z. Kai, T. Yuan et al., "Research of logistics center location and case analysis," Journal of Northern Jiaotong University, vol. 25, no. 5, pp. 80-82, 2001.

[17] Q. Xutao, "Research on the location of the railway logistics center based on existing railway freight station," M.S. thesis, Beijing Jiaotong University, Beijing, China, 2015.

[18] C. Guohui and L. Xiansheng, "Interactive mechanism of cointegration and causality between regional logistics and economic development," Journal of Traffic and Transportation Engineering, vol. 10, no. 5, pp. 90-96, 2010.

[19] J. Xiaofeng, Z. Xue, C. Fang et al., "Associated characteristics analysis of regional logistics and economic development based on multi-source data: the case of Yunnan Province," Economic Geography, vol. 36, no. 1, pp. 39-45, 2016.

[20] C. Weidong, "Spatial pattern and location evolution of urban logistics enterprises: taking Suzhou as an example," Geographical Research, vol. 30, no. 11, pp. 1997-2007, 2011.

[21] P. Yujuan and C. Xiaoshu, "Spatial patterns and their formation mechanism of supply logistics network of wholesale markets in Guangzhou," Acta Geographica Sinica, vol. 67, no. 2, pp. 179-188, 2012.

[22] L. Guoqi, J. Fengjun, C. Yu et al., "Spatial patterns of logistics industry based on a geographic analysis of hotness degree," Progress in Geography, vol. 34, no. 5, pp. 629-637, 2015.

[23] Z. Ding, C. Weidong, F. Jiaojiao et al., "The spatio-temporal evolution characteristics and mechanism of Yangtze river delta city logistics development efficiency," Economic Geography, vol. 3, no. 8, pp. 103-110, 2014.

[24] Y. Shilin, C. Youhui, W. Jiawei et al., "Spatio-temporal evolution characteristics and mechanism of the port logistics system along the Yangtze river," Geographical Research, vol. 37, no. 5, pp. 925-936, 2018.

[25] T. Yun and L. Ruobing, "A brief analysis of the spatial distribution model and its changing trend of urban population in Taiwan," Academic Exchange, vol. 5, pp. 117-122, 2010.

[26] X. Wenhui, "The characteristics, influences and trends of emigration population in Taiwan," Taiwan Studies, vol. 4, pp. 72-77, 2016.

[27] C. Yi, "Analysis on population migration and its effect on industrial structure in Taiwan," Journal of Minjiang University, vol. 37, no. 3, pp. 52-59, 2016.

[28] W. Ling and J. Xiaomei, "Characteristics, experience and suggestions of Taiwan's logistics policy," Port Economy, no. 3, pp. 41-45, 2015.

[29] L. Dajie, Z. Zhihao, Z. Liyan et al., "The development of logistics performance indicator system of Taiwan," Journal of Transport Information and Safety, vol. 33, no. 3, pp. 87-94, 2015.

[30] Y. Xing, "Development and evolution of logistics policy in Taiwan and its enlightenment," Taiwan Agricultural Research, vol. 146, no. 3, pp. 6-9, 2017.

[31] "Baidu wikipedia, Taiwan," 2020, https://baike.baidu.com/ item/\%E5\%8F\%B0\%E6\%B9\%BE/122340?fr=aladdi.

[32] P. E. Pfeifer and S. J. Deutsch, "A three-stage iterative procedure for space-time modeling," Technometrics, vol. 22, no. 1, pp. 35-47, 1980. 
[33] Y. Kamarianakis and P. Prastacos, "Space-time modeling of traffic flow," Computers \& Geosciences, vol. 31, no. 2, pp. 119-133, 2005.

[34] G. Box and G. Jenkins, Time Series Analysis: Forecasting and Control, Wiley, San Francisco, CA, USA, 1976.

[35] Y. Yue and A. G.-O. Yeh, "Spatiotemporal traffic-flow dependency and short-term traffic forecasting," Environment and Planning B: Planning and Design, vol. 35, no. 5, pp. 762-771, 2008.

[36] Z. Ling, W. Jiaqiu, D. Min et al., "Spatial-temporal autocorrelation model of road network based on travelling time," Journal of Central South University (Science and Technology), vol. 43, no. 10, pp. 365-373, 2012.

[37] C. J. J. d. Figueiredo and C. M. d. M. Mota, "A classification model to evaluate the security level in a city based on GISMCDA," Mathematical Problems in Engineering, vol. 2016, Article ID 3534824, 10 pages, 2016.

[38] Chief Accounting Office of Taiwan's Executive Yuan, Taiwan Statistical Yearbook 2016, Executive Yuan, Taipei, Taiwan, 2016.

[39] L. Yisu, S. Jinming, and J. Biao, "Revision and analysis of national informatization level measurement index system," Computer System and Applications, vol. 27, no. 6, pp. 214-219, 2018. 